\title{
Efficient simulated annealing based solution approaches to the competitive single and multiple allocation hub location problems
}

\author{
Nader Ghaffarinasab ${ }^{\mathrm{a}, *}$, Alireza Motallebzadeh ${ }^{\mathrm{b}}$, Younis Jabarzadeh ${ }^{\mathrm{b}}$, Bahar Y. Kara ${ }^{\mathrm{c}}$ \\ a Department of Industrial Engineering, University of Tabriz, Tabriz, Iran \\ ${ }^{\mathrm{b}}$ Faculty of Economics, Management and Business, University of Tabriz, Tabriz, Iran \\ ${ }^{\mathrm{c}}$ Department of Industrial Engineering, Bilkent University, Ankara, Turkey
}

\section{A R T I C L E I N F O}

\section{Article history:}

Received 3 October 2016

Revised 20 September 2017

Accepted 21 September 2017

Available online 22 September 2017

\section{Keywords:}

Hub location

Market competition

Mathematical formulation

Simulated annealing

\begin{abstract}
A B S T R A C T
Hub location problems (HLPs) constitute an important class of problems in logistics with numerous applications in passenger/cargo transportation, postal services, telecommunications, etc. This paper addresses the competitive single and multiple allocation HLPs where the market is assumed to be a duopoly. Two firms (decision makers) sequentially decide on the configuration of their hub networks trying to maximize their own market shares. The customers choose one firm based on the cost of service provided by these firms. Mathematical formulations are presented for the problems of the first and second firms (the leader and the follower, respectively) and Simulated Annealing (SA) based solution algorithms are proposed for solving these problems both in single and multiple allocation settings. Extensive computational experiments show the capability of the proposed solution algorithms to obtain the optimal solutions in short computational times. Some managerial insights are also derived based on the obtained results.
\end{abstract}

(c) 2017 Elsevier Ltd. All rights reserved.

\section{Introduction}

Hubs are special facilities that serve as switching, transshipment, and sorting points in many-to-many distribution systems. Instead of serving each origin-destination (O/D) pair directly, hub facilities concentrate flows in order to take advantage of economies of scale. Flows from the same origin with different destinations are consolidated on their route to the hub and are combined with flows that have different origins but the same destination. The consolidation takes place on the route from the origin to the hub and from the hub to the destination as well as between hubs. The hub location problem (HLP) is concerned with locating the hub facilities and allocating the demand nodes to the hubs in order to route the traffic between O/D pairs (Alumur and Kara, 2008).

Regarding the way the non-hub nodes are allocated to the hubs, there are two basic types of hub networks: single allocation and multiple allocation. In single allocation networks, all the incoming and outgoing traffic to and from any non-hub node is routed through a single hub, whereas in multiple allocation networks, each non-hub node can receive and send flow through more than one hub. Fig. 1 illustrates examples of single and multiple allocation hub networks. In both cases, four out of 14 nodes are selected

\footnotetext{
* Corresponding author.

E-mail addresses: ngnasab@tabrizu.ac.ir, ngnasab@gmail.com (N. Ghaffarinasab).
}

as hub facilities and act as consolidation and dissemination points for the traffic flows in the network.

From an applicability point of view, both single and multiple allocation networks are used in practice. For example, passenger airline networks typically have multiple allocation because there are flights from some non-hub cities to several or all of an airline's hubs, whereas less-than-truckload (LTL) trucking networks may have each non-hub node (i.e., end-of-line terminal) assigned to a single break-bulk terminal (i.e., hub). Similarly, some telecommunication networks employ the single allocation setting to reduce the cost of constructing the network, and others allow or require multiple allocation, as for example to increase reliability and/or provide backups (Campbell and O'Kelly, 2012).

In most of the studies in the literature of the HLP, the market is assumed to be a monopoly, i.e., one firm (decision maker) decides on the configuration of its hub network in order to optimize some objective of interest. However, in real world applications, there may be competitors present in the market whose decisions would definitely affect the level of success of the other firms. In this study, we consider a duopoly market where there are two operating firms. The decision maker who makes the initial location decisions is called the leader and the other one is named as the follower. We first consider a simpler case where the leader has already configured its network without being aware of the follower's upcoming entrance to the market. In this case, the problem is formulated as a single level MIP model from the follower's perspec- 


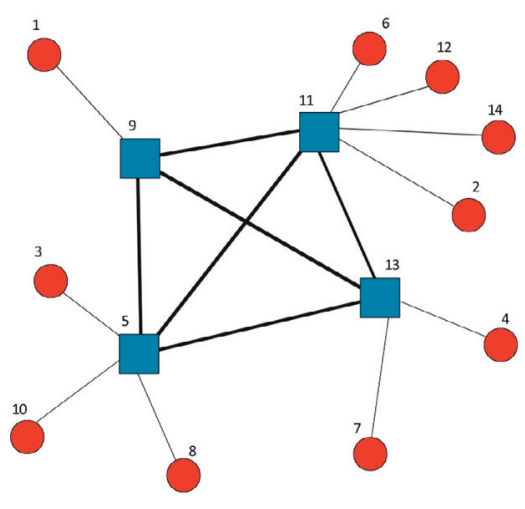

(a) single allocation

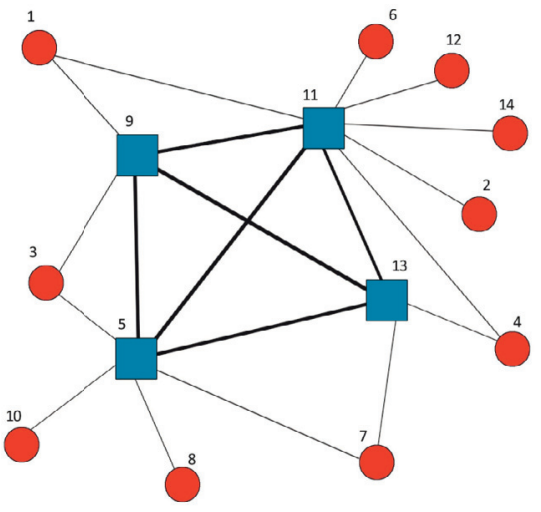

(b) multiple allocation

Fig. 1. Examples of single and multiple allocation hub networks.

tive. Then, we consider a Stackelberg game where competitors are aware of each other and the leader locates his/her hubs in anticipation of the follower's upcoming action which aims at optimally locating its hubs, based on the known decisions made by leader. Therefore, the leader seeks to locate its hubs so that its market share is maximized after the follower best locates its hubs. The problems for both single and multiple allocation networks are formulated as bilevel programming models where the upper (leader) and lower (follower) problems are MIPs.

The underlying hub networks considered in our work are designed based on the assumption of a complete network between the installed hubs equipped with efficient means of transport that allow a flow-independent discount factor to be applied to the inter-hub transportation costs. It is also assumed that the network nodes and the connecting links are uncapacitated and direct shipments are not allowed between the non-hub nodes. We remark here that, especially for single allocation case with large number of installed hubs, some of the spoke links, which are not discounted, may also have high volume of flow (Campbell, 2013). However, we do not address this issue in the current paper as our main motivation is to develop efficient metaheuristic algorithms for solving large scale instances of the competitive hub location problems under classical settings. Here we mainly focus the application towards intermodal transportation where discounts are applicable to the inter-hub links regardless of the flow volumes and due to the use of a cheaper mode of transportation (e.g., rail or maritime transportation). One sholud also note that, in case of passenger transportation, the issue of larger flows on the spoke links can partly be alleviated by separating the 0 -stop and 1-stop flows from the 2-stop flows and scheduling them separately (Campbell, 2013).

HLPs constitute a difficult class of NP-hard combinatorial optimization problems (Contreras et al., 2011). Moreover, in case of the single allocation HLP, given a fixed set of locations for the hubs, the allocation part of the problem is still NP-hard (Kara, 1999). For this reason, developing efficient solution algorithms capable of solving the problem instances of large sizes is of utmost practical importance.

The main contribution of this paper can be stated as follows. We address the competitive hub location problem under both single and multiple allocation settings. As mentioned earlier, both the allocation schemes are frequently used in practice. Therefore, studying the problem under both allocation settings is of great importance. We propose single level and bilevel MIP formulations to model the problems from the follower's and the leader's perspectives, respectively. In order to solve the proposed models, we develop four efficient solution algorithms based on Simulated Annealing (SA) that are able to solve large scale instances of the problem within short computational times. Extensive computational experiments are conducted to show the effectiveness of the proposed algorithms as well as to study the effect of different input parameters such as the number of installed hubs and the discount factor value. Furthermore, we extend the problems to accommodate more general capture mechanisms that allow the competing firms to partially capture the O/D demands in addition to the classical binary (all-or-nothing) capture mechanism. Finally, some managerial insights are derived based on the results obtained from the conducted experiments.

The remainder of this paper is organized as follows. The next section discusses the relevant literature for the problem at hand. In Section 3, we will present new bilevel MIP model formulations for the competitive hub location problem on behalf of the competitors for both single and multiple allocation networks. The proposed SA based solution algorithms are presented in Section 4. Computational experiments and corresponding results are presented in Section 5. Finally, Section 6 provides conclusions and some outlooks for future research.

\section{Literature review}

Study of the HLP began with the pioneering work of O'Kelly (1986). The first quadratic mathematical formulation of single allocation $p$-hub median problem is presented in O'Kelly (1987). Linear integer programming formulations for different versions of the HLP such as the $p$-hub median problem, the uncapacitated hub location problem, the $p$-hub center problem, and the hub covering problem are proposed by Campbell (1994). The interested readers may refer to Alumur and Kara (2008), Campbell and O'Kelly (2012), Farahani et al. (2013) and Contreras (2015) as recent surveys on the HLP.

Although the competitive facility location problem has been widely studied in the literature (see Drezner et al., 2015; Eiselt et al., 2015; Fernández et al., 2017; Kress and Pesch, 2012; Kucukaydin et al., 2012 and references therein), there is a limited number of works published in the filed of competitive hub location problem. The first work on the competitive HLP is done by Marianov et al. (1999) where they formulate the follower's problem, trying to maximize its own market share, given a set of existing hubs for the leader. Their model allows partial captures by the follower depending on the cost of services. Wagner (2008) tackles the same problem with a different capture paradigm where 
the follower gets nothing in case of equal cost. He also proposes a more efficient formulation for the problem. Sasaki and Fukushima (2001) study the competitive HLP on a plane where a large leader firm competes with several medium firms to maximize its own profit. The problem is modeled as a bilevel program where each firm locates one hub and logit functions are used to model the customer preferences that affect the proportional captures. Based on the same idea, Sasaki (2005) studies the competitive HLP in a discrete environment. The same capture rule as in Sasaki and Fukushima (2001) is used where the leader and follower respectively locate $p$ and $r$ hubs on a network and each route contains only one hub.

Adler and Smilowitz (2007) analyze the hub network alliances and mergers in the airline industry under competition. Their research combines profit-maximizing objectives to cost-based network design formulations within a game theoretic framework that enables merging airlines to choose appropriate international hubs for their integrated network. In another competitive HLP, Eiselt and Marianov (2009) address a conditional $p$-hub location problem with attraction functions where an entrant airline transportation firm is assumed to enter a competitive market and the customers are assumed to choose an airline depending on a combination of factors such as flying time and travel fare based on gravity-like utility functions.

Gelareh et al. (2010) propose an MIP model for a competitive hub network design considering competition between a newcomer liner service provider and an existing dominating operator, both operating on hub networks. The authors only address the follower's problem and propose a Lagrangian decomposition method together with a primal bound generation procedure for solving it. Lin and Lee (2010) study a competition game on hub network design and determine a hub network for each of all carriers in the oligopolistic market based on the long-term Cournot-Nash equilibrium steady state. Lüer-Villagra and Marianov (2013) address a competitive HLP in which location and pricing decisions are made by an entrant firm entering to a market where some other firm has already been operating. Customer preferences are modeled using logit function resulting in a nonlinear model maximizing the profit of the entrant firm. Sasaki et al. (2014) consider a competitive hub arc location problem under Stackelberg competition. Rather than locating hub facilities, they locate hub arcs in the network. They model the problem as a bilevel program in which the leader and the follower respectively locate $p$ and $r$ hub arcs to maximize their own revenues.

Mahmutogullari and Kara (2016) consider a competitive HLP based on Stackelberg competition where the market is assumed to be a duopoly. Two firms decide on locations of their hubs and then customers choose one firm with respect to cost of provided service. They term the follower's problem as $\left(r \mid X_{p}\right)$ hub-medianoid and the leader's problem as $(r \mid p)$ hub-centroid problem. They only consider the problems under the multiple allocation assumption and propose MIP models for them. Furthermore, they assume a binary capture mechanism where each O/D demand can be captured by either the leader or the follower. In order to solve the bilevel $(r \mid p)$ hub-centroid problem, they propose an exact solution algorithm based on enumeration and solve the problem for a network of 81 nodes with up to 5 installed hubs by the leader and the follower.

Although integer programming optimization approaches are used to solve various types of HLP in small sizes, larger instances are usually solved by heuristic or metaheuristic procedures. In fact, development of metaheuristic algorithms has helped many real world applications, in which optimal or near-optimal solutions can be obtained in less computational time. Some authors have tackled the multiple allocation HLPs using heuristic and metaheuristic algorithms (see Boland et al., 2004; Campbell, 1996; Chahar- sooghi et al., 2017; Ernst and Krishnamoorthy, 1998; Lüer-Villagra and Marianov, 2013; Marianov et al., 1999 as some examples).

In case of the single allocation HLP, the number of proposed metaheuristic algorithms are much larger. O'Kelly (1987) proposes two heuristic allocation procedures for solving the uncapacitated single allocation $p$-hub median problem (USApHMP). A tabu search (TS) heuristic is proposed for the USApHMP in SkorinKapov and Skorin-Kapov (1994). Abdinnour-Helm and Venkataramanan (1998) present a branch and bound procedure and a genetic algorithm (GA) to solve the uncapacitated single allocation hub location problem (USAHLP). Ernst and Krishnamoorthy (1996) develop a simulated annealing (SA) heuristic for the same problem and show that it is comparable, in both solution quality and computational time, to the TS heuristic in SkorinKapov and Skorin-Kapov (1994). In another work, Ernst and $\mathrm{Kr}$ ishnamoorthy (1999) propose heuristic algorithms for solving the capacitated single allocation HLP based on SA and random descent heuristic. Abdinnour-Helm presents an SA heuristic for the USApHMP (Abdinnour-Helm, 2001). Topcuoglu et al. (2005) develop a GA for the USAHLP. Chen (2007) proposes another heuristic for this problem based on an SA embedded with a tabu list and some improvement procedures. Silva and Cunha (2009) present three variants of a simple and efficient multi-start TS heuristic as well as a two-stage integrated TS heuristic to solve USAHLP. Calik et al. (2009) propose a TS heuristic for the single allocation hub covering problem over incomplete hub networks. Jabalameli et al. (2012) develop an SA heuristic for solving the uncapacitated single allocation $p$-hub maximal covering problem. Abyazi-Sani and Ghanbari (2016) present a TS based heuristic for the USAHLP. More recently, Silva and Cunha (2017) propose an efficient TS algorithm for solving the uncapacitated single allocation p-hub maximal covering problem.

\section{Mathematical formulation}

Let $G=(N, E)$ be a network, where $N$ is the set of nodes and $E$ is the set of edges such that $E \subseteq N \times N$. Assume $H \subseteq N$ be a subset of nodes that is available for locating hubs. For all $i, j \in N$, let $w_{i j}$ and $c_{i j}$ denote respectively the amount of flow originated at node $i$ and destined to node $j$, and the transportation cost of a unit flow from node $i$ to node $j$. Transportation costs on the inter-hub connections are discounted by a constant factor $\alpha(0 \leq \alpha \leq 1)$ and the number of hubs to be located by the leader and follower are denoted by $p$ and $r$, respectively. It is assumed that both the leader and follower have complete information about the game and will act rationally. Each O/D flow in the network is captured by either the leader or the follower based on the unit transportation cost from its origin to its destination. A customer prefers the follower if the cost of service provided by the follower is strictly better than that of the leader. Otherwise, the demand is captured by the leader. In case of equal costs, ties are broken in favor of the leader as the customer has no incentive to change the current position.

In the remainder of this section, mathematical formulations for the leader's and the follower's problems are proposed under both the multiple and single allocation settings. The notations and definitions for the multiple allocation case are mostly borrowed from Mahmutogullari and Kara (2016) as we address similar problems and we want the terminology to be consistent in the competitive HLP literature.

\subsection{Multiple allocation models}

Let us assume that the leader has already opened its hubs at a subset of nodes $X_{p}=\left\{x_{1}, x_{2}, \ldots, x_{p}\right\}, X_{p} \subseteq H$, and is serving the market with these hubs. For every node pair $i$ and $j$, the cost of service provided by the leader, denoted by $\beta_{i j}$, can be calculated 
as:

$\beta_{i j}=\min _{k, m \in X_{p}}\left\{c_{i k}+\alpha c_{k m}+c_{m j}\right\} \quad \forall i, j \in N$

Assume now that the follower enters the market and establishes its own hubs on a subset of nodes $Y_{r}=\left\{y_{1}, y_{2}, \ldots, y_{r}\right\}, Y_{r} \subseteq H$. In a similar manner, the follower's cost, denoted by $\gamma_{i j}$, for all node pairs $i$ and $j$ can be calculated as:

$\gamma_{i j}=\min _{k, m \in Y_{r}}\left\{c_{i k}+\alpha c_{k m}+c_{m j}\right\} \quad \forall i, j \in N$.

For all $i, j \in N$, the follower captures the flow $w_{i j}$ if $\gamma_{i j}<\beta_{i j}$. Therefore, total flow captured by the follower can be expressed by a mapping $f: \mathcal{P}_{p}(H) \times \mathcal{P}_{r}(H) \rightarrow[0, W]$ such that:

$f\left(X_{p}, Y_{r}\right)=\sum_{i, j \in N: \gamma_{i j}<\beta_{i j}} w_{i j}$

where $\mathcal{P}_{p}(H)$ is the set of all subsets of cardinality $p$ from $H$ and $W$ is the sum of flows over the network, i.e., $W=\sum_{i, j \in N} w_{i j}$.

Given the leader's hubs located on $X_{p}$, the multiple allocation $\left(r \mid X_{p}\right)$ hub-medianoid problem aims at locating a set of $r$ hubs that maximizes the captured demand by the follower. To model the $\left(r \mid X_{p}\right)$ hub-medianoid problem for the multiple allocation network, assume that $a_{i j}^{k m}$ is a binary covering parameter that takes the value of 1 if the flow between nodes $i$ and $j$ is captured by the follower and 0 , otherwise. In other words, with $\beta_{i j}$ defined by (1) for a given $X_{p}$, we have:

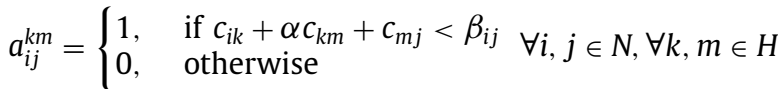

Let the variable $x_{i j k m}$ denote the fraction of flow $w_{i j}$ that is sent from node $i$ to node $j$ using the link between the hubs $k$ and $m$ by the follower. Let also the binary variable $y_{k} \in\{0,1\}$ be 1 if node $k$ is selected by the follower as a hub and 0 , otherwise. The problem consists of selecting $r$ nodes which will act as the follower's hubs and determining how the non-hub nodes will be allocated to the hubs and the flows will be routed in the network so that total captured flow by the follower is maximized. The MIP model for the multiple allocation $\left(r \mid X_{p}\right)$ hub-medianoid problem can be written as:

$F C_{M A}^{*}=\max \sum_{i \in N} \sum_{j \in N} \sum_{k \in H} \sum_{m \in H} w_{i j} a_{i j}^{k m} x_{i j k m}$

s.t.: $\sum_{k \in H} y_{k}=r$

$\sum_{k \in H} \sum_{m \in H} x_{i j k m}=1$

$\forall i, j \in N$

$\sum_{m \in H} x_{i j k m}+\sum_{m \in H \mid m \neq k} x_{i j m k} \leq y_{k}$

$\forall i, j \in N, k \in H$

$x_{i j k m} \geq 0$

$$
\forall i, j \in N, k, m \in H
$$

$y_{k} \in\{0,1\}$

$\forall k \in H$

The objective function (5) maximizes the total flow captured by the follower. Constraint (6) determines the number of hubs to be located by the follower. Constraints (7) assure that the whole flow associated with each O/D pair is routed via some hub pair. Constraints (8) state that the flows can only be routed via nodes that have been designated as hubs. (9) and (10) are positive and binary constraints, respectively.
Looking at the problem from the leader's perspective, one needs to minimize the flows captured by the follower (or equivalently maximize the flows captured by the leader) via selecting an appropriate set of hubs. In other words, the multiple allocation $(r \mid p)$ hubcentroid problem aims at selecting a set of $r$ hubs for the leader so that in the remaining scenario the follower can capture the least possible flow. To formulate the multiple allocation $(r \mid p)$ hubcentroid problem as a bilevel mathematical model, let the variables $X_{i j k m}$ and $Y_{k}$ respectively show the routing and location decisions made by the leader (corresponding to $x_{i j k m}$ and $y_{k}$ decision variables for the follower). The bilevel model for the multiple allocation $(r \mid p)$ hub-centroid problem can be written as:

$\min F C_{M A}^{*}$

s.t.: $\sum_{k \in H} Y_{k}=p$

$\sum_{k \in H} \sum_{m \in H} X_{i j k m}=1 \quad \forall i, j \in N$

$\sum_{m \in H} X_{i j k m}+\sum_{m \in H \mid m \neq k} X_{i j m k} \leq Y_{k} \quad \forall i, j \in N, k \in H$

$X_{i j k m} \geq 0 \quad \forall i, j \in N, k, m \in H$

$Y_{k} \in\{0,1\}$

$\forall i, j \in N, k \in H$

The objective function (11) minimizes the maximum total flow captured by the follower $\left(F C_{M A}^{*}\right)$ which is obtained as the optimal objective function value of the lower level problem (5)-(10). Constraint (12) forces the number of hubs opened by the leader to be equal to $p$. Constraints (13)-(16) have the same meaning for the leader as do the constraints (7)-(10) for the follower.

\subsection{Single allocation models}

We now discuss the $\left(r \mid X_{p}\right)$ hub-medianoid and $(r \mid p)$ hubcentroid problems for the single allocation case. With $X_{p}=$ $\left\{x_{1}, x_{2}, \ldots, x_{p}\right\}, X_{p} \subseteq H$, denoting the set of hubs opened by the leader, define the mapping $A_{X_{p}}^{l}: N \rightarrow X_{p}$ as the leader's assignment function consisting of ordered pairs showing the way every node in $N$ is assigned to a hub in $X_{p}$. For each node pair $i, j \in N$, let $o(i)$ and $o(j)$ denote respectively the hubs to which $i$ and $j$ are assigned according to $A_{X_{p}}^{l}$. In this case, the parameter $\beta_{i j}$ can be calculated as follows:

$\beta_{i j}=c_{i o(i)}+\alpha c_{o(i) o(j)}+c_{o(j) j} \quad \forall i, j \in N$

Now, suppose that the follower enters the market by opening its hubs on subset of nodes $Y_{r}=\left\{y_{1}, y_{2}, \ldots, y_{r}\right\}, Y_{r} \subseteq H$. Also, define $A_{Y_{r}}^{f}: N \rightarrow Y_{r}$ as the follower's assignment function showing the way every node in $N$ is assigned to a hub in $Y_{r}$. If the nodes $i$ and $j$ are respectively assigned to hubs $o^{\prime}(i)$ and $o^{\prime}(j)$ by the follower, the parameter $\gamma_{i j}$ can be calculated as follows:

$\gamma_{i j}=c_{i o^{\prime}(i)}+\alpha c_{o^{\prime}(i) o^{\prime}(j)}+c_{o^{\prime}(j) j} \quad \forall i, j \in N$

As noted before, $w_{i j}$ is captured by the follower if $\gamma_{i j}<\beta_{i j}$ for all $i, j \in N$. Given the leader's and the follower's assignment functions, $A_{X_{p}}^{l}$ and $A_{Y_{r}}^{f}$, the total flow captured by the follower can be expressed by a mapping $f: \mathcal{R}_{p}(N \times H) \times \mathcal{R}_{r}(N \times H) \rightarrow[0, W]$ such that

$f\left(A_{X_{p}}^{l}, A_{Y_{r}}^{f}\right)=\sum_{i, j \in N: \gamma_{i j}<\beta_{i j}} w_{i j}$ 
where $\mathcal{R}_{p}(N \times H)$ is the collection of all assignment functions with hubs chosen from a subset of cardinality $p$ from $H$.

The objective of the single allocation $\left(r \mid X_{p}\right)$ hub-medianoid problem is to choose a set of $r$ hubs for the follower and determine the associated assignment function that maximizes captured demand given the assignment function of the leader $\left(A_{X_{p}}^{l}\right)$. We formulate this problem based on the model proposed by Peker and Kara (2015) for the single allocation $p$-hub maximal covering problem. The $p$-hub maximal covering problem can be considered as a special case of $\left(r \mid X_{p}\right)$ hub-medianoid problem in which the covering radius for every $\mathrm{O} / \mathrm{D}$ pair is a given fixed value, whereas in the $\left(r \mid X_{p}\right)$ hub-medianoid problem, the covering radius for each $\mathrm{O} / \mathrm{D}$ pair is different from those of other pairs and is calculated based on the network configuration of the leader using Eq. (17). To model the problem, let the binary variable $y_{i k} \in\{0,1\}$ be 1 if node $i$ is allocated by the follower to hub $k$ and 0 , otherwise. Moreover, define the variable $z_{i j}$ as the fraction of flow originated from node $i$ and destined to node $j$ that is captured by the follower. Using these newly defined variables along with the $a_{i j}^{k m}$ as defined before (with $\beta_{i j}$ defined as (17)), the MIP model for the single allocation $\left(r \mid X_{p}\right)$ hub-medianoid problem can be written as:

$$
\begin{aligned}
& F C_{S A}^{*}=\max \sum_{i \in N} \sum_{j \in N} w_{i j} z_{i j} \\
& \text { s.t.: } \sum_{k \in H} y_{k k}=r \\
& \sum_{k \in H} y_{i k}=1
\end{aligned}
$$$$
\forall i \in N
$$

$y_{i k} \leq y_{k k}$

$$
\forall i \in N, k \in H
$$

$z_{i j} \leq \sum_{k \in H} a_{i j}^{k m} y_{i k}+\lambda_{i j}\left(1-y_{j m}\right) \quad \forall i, j \in N, m \in H$

$z_{i j} \geq 0$

$\forall i, j \in N$

$y_{i, k} \in\{0,1\}$

$$
\forall i \in N, k \in H
$$

The objective function (20) maximizes the total captured flow by the follower. Constraint (21) determines the number of hubs to be located by the follower. Constraints (22) imply that each node $i$ must be assigned to exactly one hub. Constraints (23) state that non-hub nodes can only be allocated to the nodes that have already established as hub nodes. Constraints (24) calculate the fraction of flow between any O/D pair $i-j$ that is captured by the follower based on the way these nodes are assigned to the installed hubs. Peker and Kara (2015) suggest to set the value of parameter $\lambda_{i j}$ to $\max _{k, m \in H}\left\{a_{i j}^{k m}\right\}$ in order to tighten the formulation. Note that based on constraints (22) every node is assigned to exactly one hub. Assuming that $o^{\prime}(i)$ and $o^{\prime}(j)$ denote respectively the hubs to which nodes $i$ and $j$ are assigned by the follower, the constraint (24) reduces either to $z_{i j} \leq a_{i j}^{o^{\prime}(i) o^{\prime}(j)}$ if $m=o^{\prime}(j)$ or to the redundant constraint $z_{i j} \leq a_{i j}^{o^{\prime}(i) m}+\lambda_{i j}$ if $m \neq o^{\prime}(j)$. Constraints (25) and (26) are standard domain constraints for the variables.

We now consider the leader's problem where he/she wants to minimize the demand captured by the follower while deciding on their hub set as well as their assignment function. To formulate a bilevel model for the single allocation $(r \mid p)$ hub-centroid problem, we define the variables $Y_{i k}$ as the assignment decisions made by the leader (corresponding to $y_{i k}$ variables for the follower). The single allocation $(r \mid p)$ hub-centroid problem can now be formulated as:

$\min F C_{S A}^{*}$

s.t.: $\sum_{k \in H} Y_{k k}=p$

$\sum_{k \in H} Y_{i k}=1$

$\forall i \in N$

$Y_{i k} \leq Y_{k k}$

$\forall i \in N, k \in H$

$Y_{i k} \in\{0,1\}$

$\forall i \in N, k \in H$

The objective function (27) minimizes the maximum total amount of flow captured by the follower $\left(F C_{S A}^{*}\right)$ calculated as the optimal objective function value of the lower level problem (20)-(26). Constraint (28) ensures that the number of hubs located by the leader is equal to $p$. Constraints (29)-(31) have the same meaning as (22),(23),(26), respectively.

The proposed bilevel models for the multiple and single allocation $(r \mid p)$ hub-centroid problems are linearized using a minimax approach and the resulting linear MIP models are presented in Appendix A.

It is known that the bilevel models are very hard to solve even for a small number of decision variables (Bard, 1998; Dempe, 2002). Therefore, we use metaheuristic solution algorithms to solve the above stated problems in reasonable time. The proposed algorithms are described in detail in the next section.

\section{Metaheuristic solution algorithm}

In this section, we describe in detail the proposed simulated annealing (SA) based metaheuristic algorithms for solving the $\left(r \mid X_{p}\right)$ hub-medianoid and $(r \mid p)$ hub-centroid problems for both the single and multiple allocation cases. SA is a metaheuristic optimization algorithm which is effective in solving combinational optimization problems. It was developed in 1953 by Metropolis et al. (1953) and was independently described by Kirkpatrick et al. (1983) and Černỳ (1985). To solve an optimization problem, the SA algorithm starts from an initial solution and consecutively moves to the new neighboring solutions via algorithm loops. If the new solution is better than the current solution in terms of the value of objective function, the current solution is replaced by the new one. Otherwise, the algorithm accepts the new solution with a probability $\exp ^{-\Delta E / T}$ if the problem has a minimization objective (or $\exp ^{\Delta E / T}$ if the problem has a maximization objective), where $\Delta E$ is the difference of objective function values between the current solution and the new solution and $T$ is the current temperature. At each temperature, several replications run and then the temperature is reduced slowly. In the early stages where the temperature is too high, there is a high probability to accept poor solutions. In the final stages, with a gradual decrease in temperature, there will be less probability to accept a bad solution. At the end, the algorithm converges to a good solution.

\subsection{Solution representation}

We use a one-dimensional array to represent the solutions in multiple allocation problem. This array of size $p$ includes the numbers associated with the nodes that are selected as hubs. The sorting of numbers within the arrays is not important. Fig. 2 demonstrates the representation array of the solution exhibited in 


\section{\begin{tabular}{l|l|l|l}
5 & 13 & 9 & 11
\end{tabular}}

Fig. 2. Solution representation for multiple allocation problem.

\begin{tabular}{|l|l|l|l|l|l|l|l|l|l|l|l|l|l|}
\hline 0 & 0 & 0 & 0 & 1 & 0 & 0 & 0 & 1 & 0 & 1 & 0 & 1 & 0 \\
\hline
\end{tabular}

(a) hub location array

\begin{tabular}{|l|l|l|l|l|l|l|l|l|l|l|l|l|l|}
\hline 9 & 11 & 5 & 13 & 5 & 11 & 13 & 5 & 9 & 5 & 11 & 11 & 13 & 11 \\
\hline
\end{tabular}

(b) allocation array

Fig. 3. Solution representation for single allocation problem.

Fig. 1 (a) which is a generic hub network with 14 nodes where $p=4$.

Note that having known the selected hubs, for each O/D pair $i-j$, one can easily determine the paths for routing the associated flow $w_{i j}$ by solving a shortest path problem.

For the single allocation problem, we use two one-dimensional arrays of size $|N|$ to represent each solution. The first array, which is a zero-one string, is called the hub location array in which the nodes corresponding to "one" elements are chosen as hubs and the nodes corresponding to "zero" elements indicate non-hub nodes. The second array is called the allocation array which shows the hub nodes to which every node is allocated. Fig. 3 shows the representation of the solution exhibited in Fig. 1(b). As can be seen in this figure, four nodes (nodes 5, 9, 11, and 13) are established as hubs and corresponding elements in the first array in Fig. 3 take the value of 1 . In the above hub network, the node 1 (as well as node 9) is allocated to hub 9. This is illustrated in the second array where the first and ninth elements of this array take the value of 9

\subsection{Initial solution generation}

The initial solutions are generated randomly in our algorithms. To this end, we randomly select $p$ out of $|N|$ nodes as hub nodes both in multiple and single allocation problems. Furthermore, in case of single allocation network, the remaining $(|N|-p)$ nonhub nodes are randomly allocated to the selected hub nodes. This procedure for generating initial solutions not only finds a solution quickly but it also produces diverse starting points which can help the algorithm produce high quality solutions in different runs by not getting trapped in local optima.

\subsection{Neighborhood structures}

We define and use two different operators for generating neighboring solutions for the multiple allocation SA algorithms. The first operator is called "Swap_One_Hub" and the second one is called "Swap_Two_Hubs". Both of the operators use a current solution to generate a random neighboring solution as explained below:

- Swap_One_Hub: This operator is used to alter one of the hubs in the solutions. First, we randomly select a hub node and a non-hub node. Then the selected hub node becomes non-hub and the selected non-hub node becomes hub.

- Swap_Two_Hubs: This operator is quite similar to the former one except for that in this case two hub nodes and two nonhub nodes are selected randomly and the above procedure is repeated for each pair of hub and non-hub nodes. When applied to a solution, this operator generates more diverse neighbors than the previous one.

Having altered the set of open hubs using either of the two above mentioned operators, the allocations of flows are then determined based on the new set of hubs by solving a shortest path problem for each O/D pair $i-j$, as mentioned before.

For single allocation SA algorithm, we use three other operators for generating neighboring solutions, namely the "Swap_Hub", the "Nearest_Allocation", and the "Reallocate_NonHub" operators. "Swap_Hub" is used to generate random neighbors from the current solution. "Nearest_Allocation" is used to allocate the non-hub nodes to their nearest open hubs immediately after the "Swap_Hub" operator is applied, whereas "Reallocate_NonHub" is used to perform local search on the newly generated neighbors to improve their assignment parts.

- Swap_Hub: In this move, a randomly selected hub node becomes non-hub and a randomly selected non-hub node becomes hub. The new non-hub node as well as the nodes previously allocated to it are then allocated to other existing hubs based on nearest distance policy.

- Nearest_Allocation: Based on this operator, which is originally proposed by O'Kelly (1987), for a given set of hub nodes, each non-hub node is allocated to its nearest open hub.

- Reallocate_NonHub: This operator changes the allocation of a randomly selected non-hub node to a hub node other than its current hub.

\subsection{Parameters used in the SA procedure}

The proposed SA algorithms use six input parameters, namely $T_{0}, T_{F}, \delta_{1}, \delta_{2}, R$, and $N_{e} . T_{0}$ and $T_{F}$ represent the initial and final temperatures, respectively. $\delta_{1}$ is used in the $(r \mid p)$ hub-centroid problems as cooling rate that controls the colling process in the algorithm, whereas $\delta_{2}$ is used as the cooling rate in the $\left(r \mid X_{p}\right)$ hubmedianoid problems. $R(0<R<1)$ denotes the probability with which the first operator is used at each temperature of the SA algorithm in multiple allocation problems (medianoid and centroid). The second operator is thus used with probability $1-R$. Finally, $N_{e}$ denotes the number of iterations the search proceeds at a particular temperature which is used only in single allocation problems (medianoid and centroid).

\subsection{The overall SA algorithms}

To solve the multiple allocation $\left(r \mid X_{p}\right)$ hub-medianoid problem, we assume that the leader has already located its hubs based on the solution of the uncapacitated multiple allocation $p$-hub median problem (UMApHMP). We start our algorithm by generating an initial solution $Y_{r}$ for the follower and setting the initial temperature to $T_{0}$. $Y_{\text {best }}$ denotes the best solution found so far and $f_{\text {best }}$ denotes the corresponding objective function value. At each temperature, we generate a new solution $Y_{r}^{\prime}$ based on the current solution using either of the two operators presented in Section 4.3. To generate a neighboring solution, a random number $\rho$ is generated from the interval $[0,1]$ and if this number is larger than the threshold value of the $R$, we use the "Swap_One_Hub" operator and otherwise, we use "Swap_Two_Hubs" operator. Subsequently, the objective value of the new solution $f\left(X_{p}, Y_{r}^{\prime}\right)$ is calculated. We define $\Delta E$ as the difference between the objective values of the new and current solutions, i.e., $\Delta E=f\left(X_{p}, Y_{r}^{\prime}\right)-f\left(X_{p}, Y_{r}\right)$. If $\Delta E>0$, we update the current solution as $Y_{r} \leftarrow Y_{r}^{\prime}$. If the objective $f\left(X_{p}, Y_{r}^{\prime}\right)$ of the new solution $Y_{r}^{\prime}$ is even larger than the best objective $f_{\text {best }}$, we set $f_{\text {best }} \leftarrow f\left(X_{p}, Y_{r}^{\prime}\right)$ and $Y_{\text {best }} \leftarrow Y_{r}^{\prime}$. Otherwise, if $\Delta E \leq 0$, we generate another random number $\rho$ from the interval [0,1]. If $\rho$ is larger than $\exp (\Delta E / T)$, we update the current solution as $Y_{r} \leftarrow Y_{r}^{\prime}$. In other words, we accept the solutions of worse quality with probability $\exp (\Delta E / T)$ to help the algorithm not get trapped in local optima. Subsequently, we reduce the temperature at each iteration according to the formula $T=\delta \times T$. The algorithm 
is terminated when the current temperature $T$ drops below the prespecified final temperature $T_{F}$.

The SA algorithm for the multiple allocation $(r \mid p)$ hub-centroid problem is more complex than that of the $\left(r \mid X_{p}\right)$ hub-medianoid problem as we aim at optimizing the leader's objective within a bilevel solution framework. In this case, we have a main SA for optimizing the leader's decisions and a lower level SA for the follower's decisions. At each iteration of the main SA, where a new solution is generated for the leader, the lower level SA is run on behalf of the follower to solve the $\left(r \mid X_{p}\right)$ hub-medianoid problem. In this case, we define $\Delta E=f\left(X_{p}^{\prime}, Y_{r}^{*}\left(X_{p}^{\prime}\right)\right)-f\left(X_{p}, Y_{r}^{*}\left(X_{p}\right)\right)$ in the main SA. Since the $(r \mid p)$ hub-centroid problem has a minimization objective, we accept the new solutions if $\Delta E<0$. Furthermore, if the objective $f\left(X_{p}^{\prime}, Y_{r}^{*}\left(X_{p}^{\prime}\right)\right)$ of the new solution $X_{p}^{\prime}$ is smaller than best objective $f_{\text {best }}$, we set $f_{\text {best }} \leftarrow f\left(X_{p}^{\prime}, Y_{r}^{*}\left(X_{p}^{\prime}\right)\right)$ and $X_{\text {best }} \leftarrow X_{p}^{\prime}$. Otherwise, if $\Delta E \geq 0$, we accept this solution of inferior quality with probability $\exp (-\Delta E / T)$.

The pseudo-codes of the proposed SA algorithms for the multiple allocation $\left(r \mid X_{p}\right)$ hub-medianoid and the multiple allocation $(r \mid p)$ hub-centroid problems are illustrated in Algorithms 1 and 2, respectively.

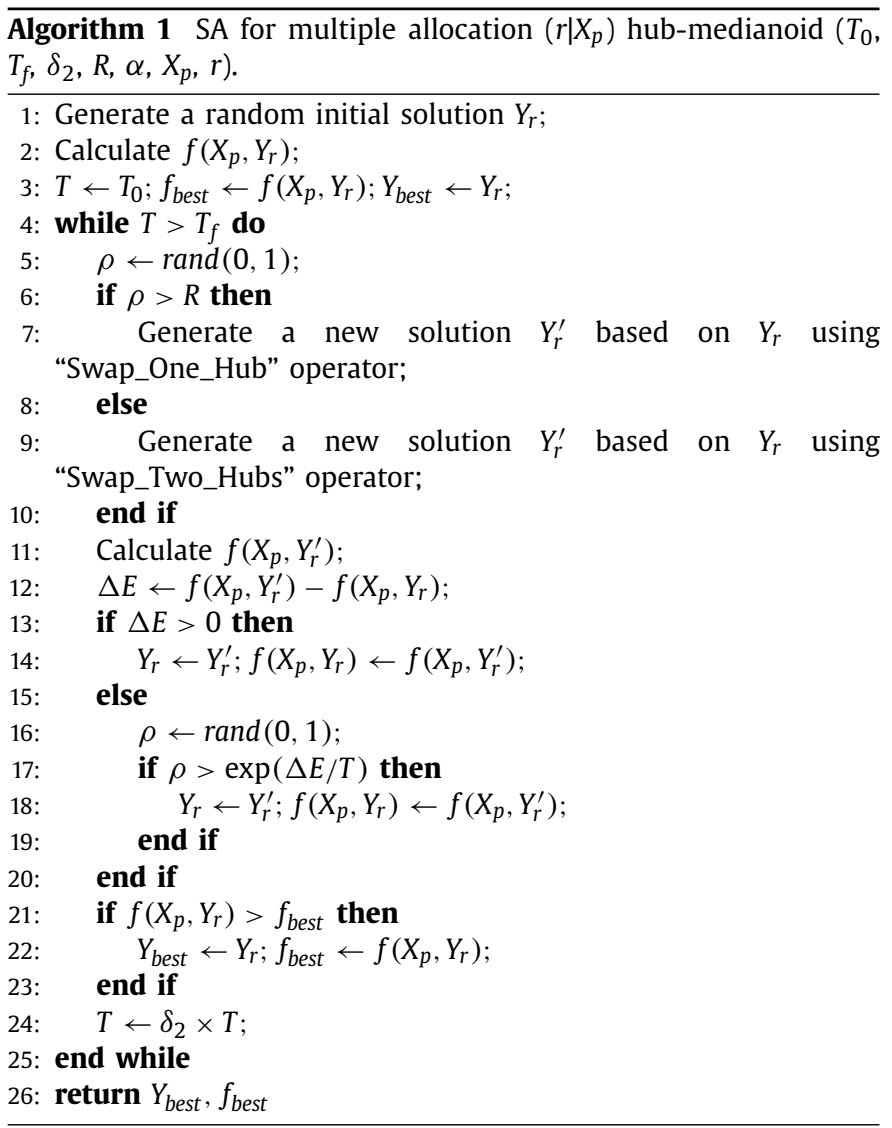

The proposed SA algorithms for the single allocation $\left(r \mid X_{p}\right)$ hubmedianoid and $(r \mid p)$ hub-centroid problems are in general very similar to their multiple allocation counterparts. However, the solution representation scheme and the employed neighborhood structures are different that those of the multiple allocation problems, as discussed earlier. Furthermore, in case of the single allocation problems, number of neighboring solutions generated at each temperature is $N_{e}$ after which a local search is performed on the best found solution based on the "Reallocate_NonHub" operator. The pseudo-codes of the proposed SA algorithms for the single allocation $\left(r \mid X_{p}\right)$ hub-medianoid and the single allocation $(r \mid p)$ hubcentroid problems are shown in Algorithms 3 and 4, respectively. $\overline{\text { Algorithm } 2 \text { SA for multiple allocation }(r \mid p) \text { hub-centroid }\left(T_{0}, T_{f}\right.}$, $\left.\delta_{1}, R, \alpha, p, r\right)$.

1: Generate a random initial solution $X_{p}$;

2: Get $Y_{r}^{*}$ and $f\left(X_{p}, Y_{r}^{*}\right)$ by solving the $\left(r \mid X_{p}\right)$ hub-medianoid problem using Algorithm 1;

3: $T \leftarrow T_{0} ; f_{\text {best }} \leftarrow f\left(X_{p}, Y_{r}^{*}\right) ; X_{\text {best }} \leftarrow X_{p}$;

: while $T>T_{f}$ do

$\rho \leftarrow \operatorname{rand}(0,1)$;

if $\rho>R$ then

Generate a new solution $X_{p}^{\prime}$ based on $X_{p}$ using

"Swap_One_Hub" operator;

8: $\quad$ else

9: Generate a new solution $X_{p}^{\prime}$ based on $X_{p}$ using

"Swap_Two_Hubs" operator;

10: $\quad$ end if

11: $\quad$ Get $Y_{r}^{*}$ and $f\left(X_{p}^{\prime}, Y_{r}^{*}\right)$ by solving $\left(r \mid X_{p}\right)$ hub-medianoid problem using Algorithm 1;

12: $\Delta E \leftarrow f\left(X_{p}^{\prime}, Y_{r}^{*}\right)-f\left(X_{p}, Y_{r}^{*}\right)$;

13: if $\Delta E<0$ then

14: $\quad X_{p} \leftarrow X_{p}^{\prime} ; f\left(X_{p}, Y_{r}^{*}\right) \leftarrow f\left(X_{p}^{\prime}, Y_{r}^{*}\right)$;

15: $\quad$ else

16: $\quad \rho \leftarrow \operatorname{rand}(0,1)$;

17: $\quad$ if $\rho<\exp (-\Delta E / T)$ then

18: $\quad X_{p} \leftarrow X_{p}^{\prime} ; f\left(X_{p}, Y_{r}^{*}\right) \leftarrow f\left(X_{p}^{\prime}, Y_{r}^{*}\right)$;

19: $\quad$ end if

20: $\quad$ end if

21: $\quad$ if $f\left(X_{p}, Y_{r}^{*}\right)<f_{\text {best }}$ then

22: $\quad X_{\text {best }} \leftarrow X_{p} ; f_{\text {best }} \leftarrow f\left(X_{p}, Y_{r}^{*}\right)$;

23: $\quad$ end if

24: $\quad T \leftarrow \delta_{1} \times T$;

5: end while

return $X_{\text {best }}, f_{\text {best }}$

Table 1

Test instances used for $\left(r \mid X_{p}\right)$ hub-medianoid problem.

\begin{tabular}{llll}
\hline Data set & $\mathrm{CAB}$ & $\mathrm{TR}(r, p \leq 5)$ & $\mathrm{TR}(r, p \geq 6)$ \\
\hline$p$ & $2,3,4$, and 5 & $2,3,4$, and 5 & $6,8,10,12$, and 14 \\
$r$ & $2,3,4$, and 5 & $2,3,4$, and 5 & $6,8,10,12$, and 14 \\
$\alpha$ & 0.6 and 0.8 & $0.6,0.8$, and 0.9 & $0.6,0.8$, and 0.9 \\
\hline
\end{tabular}

\section{Computational experiments}

In order to test the efficiency of the proposed SA algorithms, we use two data sets from the literature of the HLP: the CAB and the TR data sets. The CAB data set introduced by O'Kelly (1987) is based on the airline passenger interactions between 25 US cities in 1970 evaluated by the Civil Aeronautics Board (CAB). This data set has been used by most of the hub location researchers in the literature. To solve the problem on the CAB data set, the parameter $\alpha$ is considered at two levels as $\alpha \in\{0.6,0.8\}$. The second data set that is used in our computational experiments is the TR data set (Tan and Kara, 2007) which is based on the cargo flows between 81 cities of Turkey where only 22 of these cities are candidate nodes for location of hubs $(|H|=22)$. The parameter $\alpha$ is considered at three levels as $\alpha \in\{0.6,0.8,0.9\}$ for the TR data set. The proposed SA algorithms are implemented in Microsoft Visual C\# 2013 (version 5.0). Also, the proposed mathematical models for the single and multiple allocation $\left(r \mid X_{p}\right)$ hub-medianoid problems are solved independently using CPLEX version 12.6. All the experiments have been run on a computer with Intel(R) Core(TM) i3$3220 \mathrm{CPU}$ of $3.30 \mathrm{GHz}$ and 16GB of RAM, using the Microsoft Windows 7 operating system. Table 1 summarizes all test instances used in the computational study of the $\left(r \mid X_{p}\right)$ hub-medianoid problem for multiple and single allocation networks. 
Algorithm 3 SA for single allocation $\left(r \mid X_{p}\right)$ hub-medianoid $\left(T_{0}, T_{f}, \delta_{2}, N_{e}, \alpha, A_{X_{p}}^{l}, r\right)$.

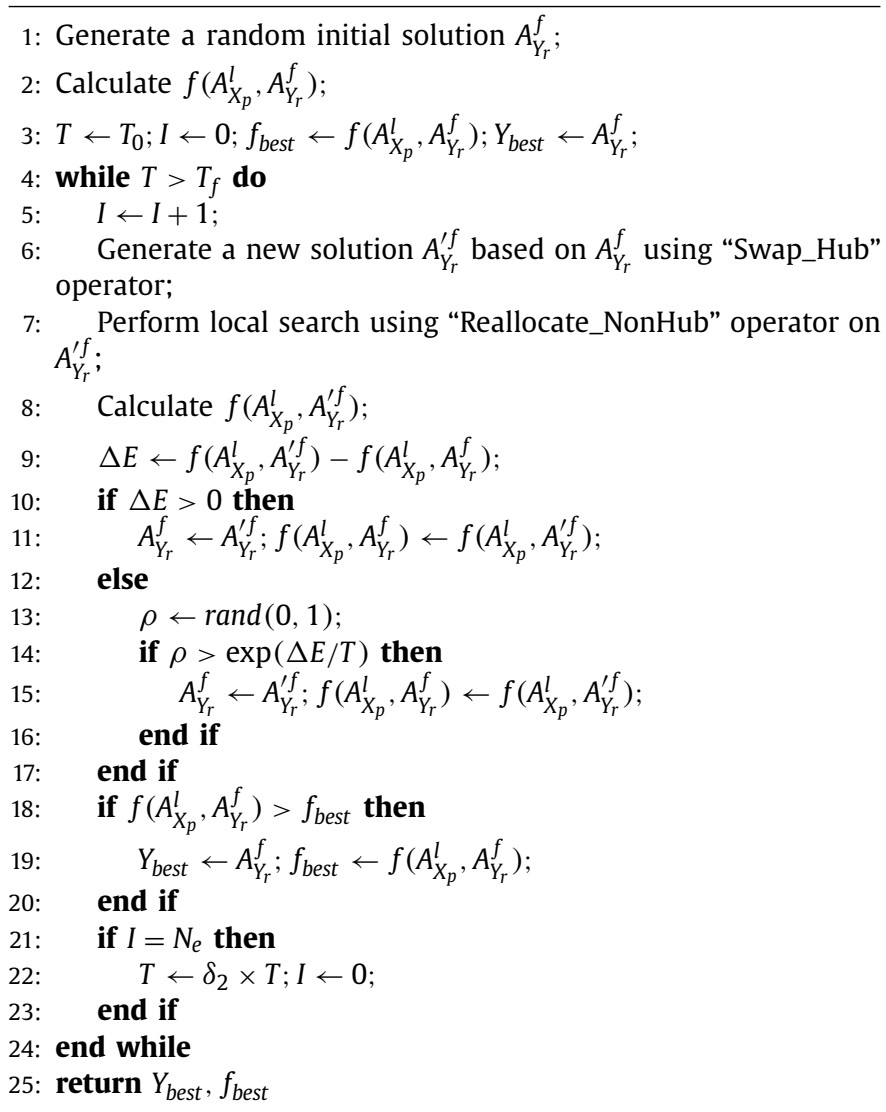

Table 2

Parameters of the SA algorithms.

\begin{tabular}{lllllll}
\hline & $T_{0}$ & $T_{f}$ & $\delta_{1}$ & $\delta_{2}$ & $N_{e}$ & $R$ \\
\hline Multiple allocation & 100 & 1 & 0.98 & 0.99 & - & 0.67 \\
Single allocation & 20,000 & 2000 & 0.99 & 0.90 & 25 & - \\
\hline
\end{tabular}

For the multiple allocation $(r \mid p)$ hub-centroid problem, all the test instances shown in Table 1 are solved. However, for the single allocation case, only the instances with $r, p \leq 5$ for the TR data set are solved. Furthermore, for the latter case, some small instances from the $C A B$ data set with $|N| \in\{10,15\}$ and $p, r \in\{2,3\}$ are solved using the proposed SA algorithm to compare its performance with that of an enumeration algorithm adapted from Mahmutogullari and Kara (2016).

The parameters of the proposed SA algorithms are tuned by setting a good trade-off between time and quality of the solutions. In an initial set of experiments, different combinations of parameters were tested on a large number of test instances and the values reported in Table 2 have been selected as the best values which lead to high-quality solutions in short CPU times for multiple and single allocation versions of the problem.

A comprehensive set of computational experiments are conducted using the above mentioned test problems to show the efficiency of the proposed SA algorithms and the results are reported in the following sub-sections. For each problem instance, we have run the SA algorithm for five times and the best solutions obtained are reported.

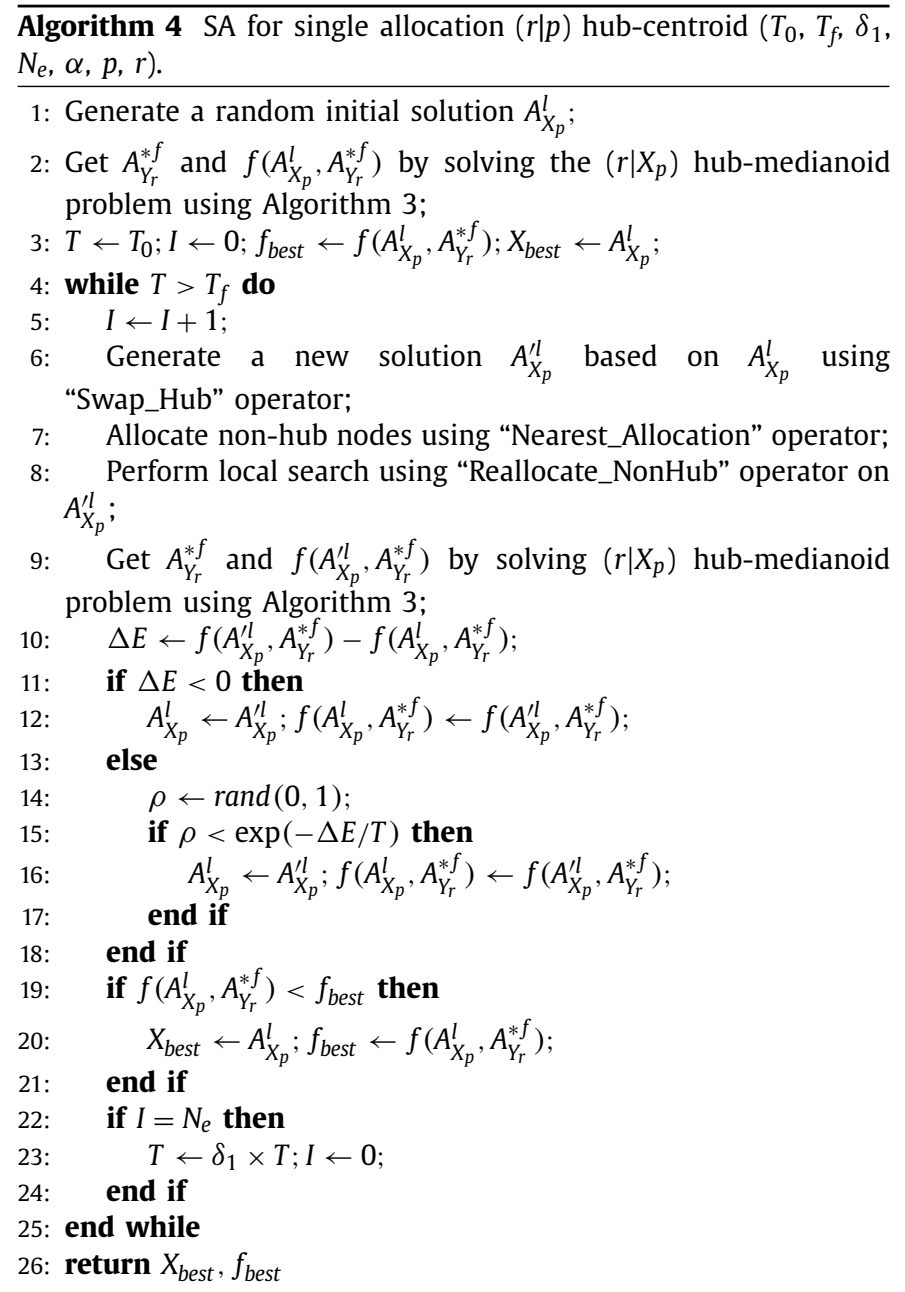

\subsection{Results for the multiple allocation case}

Table 3 shows the results obtained by solving the multiple allocation $\left(r \mid X_{p}\right)$ hub-medianoid problem using the proposed SA algorithm as well as CPLEX based on the proposed mathematical models with the $C A B$ data set. Since the distance matrix in the $C A B$ data set (also in the TR data set) is symmetric, it is clear that if the flow $w_{i j}$ from node $i \in N$ to node $j \in N$ is captured by the follower, the flow from node $j$ to node $i$ is also captured by the follower. Therefore, to reduce the size of our model, the constraints (7)-(9) are imposed for only $i<j$ and the objective (5) is modified as $\sum_{i} \sum_{j \mid j>i} \sum_{k} \sum_{m}\left(w_{i j}+w_{j i}\right) a_{i j}^{k m} x_{i j k m}$ in our computational studies.

It is assumed that the leader has already located its hubs based on the uncapacitated multiple allocation $p$-hub median problem (UMApHMP). Different discount factor $(\alpha)$ values are shown in the first row of the table. The columns entitled $p$ and $r$ denote the number of hubs which are opened by the leader and the follower, respectively. The next two columns show the follower's capture as the optimal objective function value that has been obtained by CPLEX and the CPU time, in seconds, needed to reach that solution. Finally, the columns under the label "SA" give the best objective function obtained through solving the instances with the SA algorithm and the average CPU time for the five runs of the algorithm.

Observe that the proposed SA algorithm solves all instances to optimality within a fraction of a second which can be counted as an indication of the efficiency of the proposed SA algorithm. 
Table 3

Results for multiple allocation $\left(r \mid X_{p}\right)$ hub-medianoid problem with the CAB data set.

\begin{tabular}{|c|c|c|c|c|c|c|c|c|c|c|c|}
\hline \multicolumn{6}{|c|}{$\alpha=0.6$} & \multicolumn{6}{|c|}{$\alpha=0.8$} \\
\hline \multirow[t]{2}{*}{$p$} & \multirow[t]{2}{*}{$r$} & \multicolumn{2}{|l|}{ CPLEX } & \multicolumn{2}{|l|}{ SA } & \multirow[t]{2}{*}{$p$} & \multirow[t]{2}{*}{$r$} & \multicolumn{2}{|l|}{ CPLEX } & \multicolumn{2}{|l|}{ SA } \\
\hline & & Follower's capture & $\mathrm{CPU}(\mathrm{s})$ & Follower's capture & $\mathrm{CPU}(\mathrm{s})$ & & & Follower's capture & $\mathrm{CPU}(\mathrm{s})$ & Follower's capture & $\mathrm{CPU}(\mathrm{s})$ \\
\hline \multirow[t]{4}{*}{2} & 2 & $65.62 \%$ & 14.74 & $65.62 \%$ & 0.03 & \multirow[t]{4}{*}{2} & 2 & $65.84 \%$ & 8.82 & $65.84 \%$ & 0.03 \\
\hline & 3 & $78.25 \%$ & 17.56 & $78.25 \%$ & 0.10 & & 3 & $74.19 \%$ & 22.03 & $74.19 \%$ & 0.11 \\
\hline & 4 & $87.08 \%$ & 17.83 & $87.08 \%$ & 0.20 & & 4 & $80.69 \%$ & 25.10 & $80.69 \%$ & 0.19 \\
\hline & 5 & $92.38 \%$ & 16.05 & $92.38 \%$ & 0.22 & & 5 & $87.14 \%$ & 18.35 & $87.14 \%$ & 0.21 \\
\hline \multirow[t]{4}{*}{3} & 2 & $30.49 \%$ & 26.60 & $30.49 \%$ & 0.04 & \multirow[t]{4}{*}{3} & 2 & $29.18 \%$ & 24.99 & $29.18 \%$ & 0.05 \\
\hline & 3 & $45.13 \%$ & 24.56 & $45.13 \%$ & 0.14 & & 3 & $42.92 \%$ & 22.58 & $42.92 \%$ & 0.13 \\
\hline & 4 & $53.69 \%$ & 22.13 & $53.69 \%$ & 0.23 & & 4 & $52.83 \%$ & 20.57 & $52.83 \%$ & 0.25 \\
\hline & 5 & $62.02 \%$ & 23.93 & $62.02 \%$ & 0.27 & & 5 & $60.14 \%$ & 22.47 & $60.14 \%$ & 0.27 \\
\hline \multirow[t]{4}{*}{4} & 2 & $18.89 \%$ & 27.72 & $18.89 \%$ & 0.07 & \multirow[t]{4}{*}{4} & 2 & $21.06 \%$ & 24.48 & $21.06 \%$ & 0.07 \\
\hline & 3 & $28.39 \%$ & 30.68 & $28.39 \%$ & 0.17 & & 3 & $32.69 \%$ & 21.92 & $32.69 \%$ & 0.18 \\
\hline & 4 & $37.73 \%$ & 33.16 & $37.73 \%$ & 0.30 & & 4 & $42.10 \%$ & 23.47 & $42.10 \%$ & 0.34 \\
\hline & 5 & $46.18 \%$ & 25.76 & $46.18 \%$ & 0.30 & & 5 & $48.60 \%$ & 25.25 & $48.60 \%$ & 0.39 \\
\hline \multirow[t]{4}{*}{5} & 2 & $18.64 \%$ & 27.97 & $18.64 \%$ & 0.09 & \multirow[t]{4}{*}{5} & 2 & $18.19 \%$ & 23.49 & $18.19 \%$ & 0.10 \\
\hline & 3 & $28.14 \%$ & 23.62 & $28.14 \%$ & 0.23 & & 3 & $29.12 \%$ & 20.11 & $29.12 \%$ & 0.22 \\
\hline & 4 & $35.04 \%$ & 19.22 & $35.04 \%$ & 0.32 & & 4 & $36.93 \%$ & 24.00 & $36.93 \%$ & 0.34 \\
\hline & 5 & $42.32 \%$ & 22.77 & $42.32 \%$ & 0.36 & & 5 & $44.32 \%$ & 25.48 & $44.32 \%$ & 0.43 \\
\hline \multicolumn{2}{|c|}{ Average } & $48.12 \%$ & 23.39 & $48.12 \%$ & 0.19 & \multicolumn{2}{|c|}{ Average } & $47.87 \%$ & 22.07 & $47.87 \%$ & 0.20 \\
\hline
\end{tabular}

Table 4

Results for multiple allocation $\left(r \mid X_{p}\right)$ hub-medianoid problem with the TR data set $(r, p \leq 5)$.

\begin{tabular}{|c|c|c|c|c|c|c|c|c|c|c|c|c|c|c|c|c|c|}
\hline \multicolumn{6}{|c|}{$\alpha=0.6$} & \multicolumn{6}{|c|}{$\alpha=0.8$} & \multicolumn{6}{|c|}{$\alpha=0.9$} \\
\hline \multirow[t]{2}{*}{$p$} & \multirow[t]{2}{*}{$r$} & \multicolumn{2}{|l|}{ CPLEX } & \multicolumn{2}{|l|}{ SA } & \multirow[t]{2}{*}{$\bar{p}$} & \multirow[t]{2}{*}{$r$} & \multicolumn{2}{|l|}{ CPLEX } & \multicolumn{2}{|l|}{ SA } & \multirow[t]{2}{*}{$p$} & \multirow[t]{2}{*}{$r$} & \multicolumn{2}{|l|}{ CPLEX } & \multicolumn{2}{|l|}{ SA } \\
\hline & & $\begin{array}{l}\text { Follower's } \\
\text { capture }\end{array}$ & CPU (s) & $\begin{array}{l}\text { Follower's } \\
\text { capture }\end{array}$ & $\begin{array}{l}\text { CPU } \\
(\mathrm{s})\end{array}$ & & & $\begin{array}{l}\text { Follower's } \\
\text { capture }\end{array}$ & $\mathrm{CPU}(\mathrm{s})$ & $\begin{array}{l}\text { Follower's } \\
\text { capture }\end{array}$ & $\begin{array}{l}\mathrm{CPU} \\
(\mathrm{s})\end{array}$ & & & $\begin{array}{l}\text { Follower's } \\
\text { capture }\end{array}$ & CPU (s) & $\begin{array}{l}\text { Follower's } \\
\text { capture }\end{array}$ & $\begin{array}{l}\text { CPU } \\
\text { (s) }\end{array}$ \\
\hline \multirow[t]{4}{*}{2} & 2 & $50.60 \%$ & 456.21 & $50.60 \%$ & 0.32 & \multirow[t]{4}{*}{2} & 2 & $49.95 \%$ & 538.33 & $49.95 \%$ & 0.30 & \multirow[t]{4}{*}{2} & 2 & $50.66 \%$ & 476.94 & $50.66 \%$ & 0.41 \\
\hline & 3 & $68.73 \%$ & 673.52 & $68.73 \%$ & 0.51 & & 3 & $62.48 \%$ & 878.01 & $62.48 \%$ & 0.46 & & 3 & $67.09 \%$ & 558.60 & $67.09 \%$ & 0.53 \\
\hline & 4 & $80.13 \%$ & 344.27 & $80.13 \%$ & 0.70 & & 4 & $72.47 \%$ & 568.05 & $72.47 \%$ & 0.71 & & 4 & $77.52 \%$ & 323.88 & $77.52 \%$ & 0.86 \\
\hline & 5 & $89.97 \%$ & 115.03 & $89.97 \%$ & 0.95 & & 5 & $84.88 \%$ & 132.67 & $84.88 \%$ & 1.19 & & 5 & $85.27 \%$ & 191.20 & $85.27 \%$ & 0.99 \\
\hline \multirow[t]{4}{*}{3} & 2 & $30.49 \%$ & 1873.32 & $30.49 \%$ & 0.45 & \multirow[t]{4}{*}{3} & 2 & $30.68 \%$ & 1435.06 & $30.68 \%$ & 0.45 & \multirow[t]{4}{*}{3} & 2 & $40.58 \%$ & 233.93 & $40.58 \%$ & 0.50 \\
\hline & 3 & $40.82 \%$ & 1245.46 & $40.82 \%$ & 0.66 & & 3 & $40.80 \%$ & 1667.72 & $40.80 \%$ & 0.64 & & 3 & $52.71 \%$ & 494.27 & $52.71 \%$ & 0.61 \\
\hline & 4 & $56.40 \%$ & 577.22 & $56.40 \%$ & 0.80 & & 4 & $51.43 \%$ & 959.99 & $51.43 \%$ & 0.91 & & 4 & $63.24 \%$ & 360.14 & $63.24 \%$ & 0.95 \\
\hline & 5 & $66.43 \%$ & 487.51 & $66.43 \%$ & 1.07 & & 5 & $60.66 \%$ & 560.07 & $60.66 \%$ & 1.12 & & 5 & $72.38 \%$ & 130.33 & $72.38 \%$ & 1.21 \\
\hline \multirow[t]{4}{*}{4} & 2 & $22.14 \%$ & 1389.16 & $22.14 \%$ & 0.70 & \multirow[t]{4}{*}{4} & 2 & $20.33 \%$ & 2223.10 & $20.33 \%$ & 0.73 & \multirow[t]{4}{*}{4} & 2 & $20.38 \%$ & 1465.93 & $20.38 \%$ & 0.72 \\
\hline & 3 & $33.69 \%$ & 739.71 & $33.69 \%$ & 0.93 & & 3 & $30.18 \%$ & 1897.41 & $30.18 \%$ & 0.91 & & 3 & $30.55 \%$ & 1777.43 & $30.55 \%$ & 0.88 \\
\hline & 4 & $44.79 \%$ & 949.52 & $44.79 \%$ & 1.12 & & 4 & $39.40 \%$ & 1604.90 & $39.40 \%$ & 1.17 & & 4 & $38.46 \%$ & 1316.08 & $38.46 \%$ & 1.11 \\
\hline & 5 & $55.69 \%$ & 517.45 & $55.69 \%$ & 1.49 & & 5 & $48.57 \%$ & 786.07 & $48.57 \%$ & 1.41 & & 5 & $47.40 \%$ & 565.69 & $47.40 \%$ & 1.41 \\
\hline \multirow[t]{4}{*}{5} & 2 & $15.01 \%$ & 2076.17 & $15.01 \%$ & 0.89 & \multirow[t]{4}{*}{5} & 2 & $15.72 \%$ & 1638.12 & $15.72 \%$ & 1.00 & \multirow[t]{4}{*}{5} & 2 & $16.47 \%$ & 1387.98 & $16.47 \%$ & 1.06 \\
\hline & 3 & $23.88 \%$ & 1457.35 & $23.88 \%$ & 1.09 & & 3 & $24.24 \%$ & 1618.06 & $24.24 \%$ & 1.12 & & 3 & $23.94 \%$ & 1258.68 & $23.94 \%$ & 1.13 \\
\hline & 4 & $33.97 \%$ & 528.04 & $33.97 \%$ & 1.48 & & 4 & $32.69 \%$ & 665.54 & $32.69 \%$ & 1.35 & & 4 & $33.03 \%$ & 670.19 & $33.03 \%$ & 1.37 \\
\hline & 5 & $42.20 \%$ & 397.08 & $42.20 \%$ & 1.69 & & 5 & $40.21 \%$ & 381.95 & $40.21 \%$ & 1.59 & & 5 & $41.01 \%$ & 400.56 & $41.01 \%$ & 1.55 \\
\hline \multicolumn{2}{|c|}{ Average } & $47.18 \%$ & 864.19 & $47.18 \%$ & 0.89 & \multicolumn{2}{|c|}{ Average } & $44.04 \%$ & 1097.19 & $44.04 \%$ & 0.94 & \multicolumn{2}{|c|}{ Average } & $47.54 \%$ & 725.74 & $47.54 \%$ & 0.95 \\
\hline
\end{tabular}

Also, the solution times for CPLEX using the proposed mathematical models are also acceptable for the CAB data set.

Note that since the leader decides on the location of its hubs so that the total cost is minimized (based on UMApHMP) and does not take into account the upcoming competition, the follower can capture a considerable share of market upon entrance to market. For instance, when the follower locates the same number of hubs as the leader's, i.e., $p=r$, its captured market share is larger than that of the leader. For the cases where $p \leq r$, the lost market share by the leader gets even larger. However, as $p$ increases $(p=4$ or 5 ), the follower's capture is not as much as that of the leader.

Tables 4 and 5 show the results obtained by solving the multiple allocation $\left(r \mid X_{p}\right)$ hub-medianoid problem with the TR data set for $r, p \leq 5$ and $r, p \geq 6$, respectively. Here also it is assumed that the leader has already selected its $p$ hubs based on UMApHMP. To evaluate the performance of the proposed SA algorithm on the TR data set, we have solved the instances with $r, p \leq 5$ using the proposed mathematical models using CPLEX and compared its results with those obtained by the SA algorithm. However, as the instances for $r, p \geq 6$ have been solved to optimality by Mahmutogullari and Kara (2016), for these instances the results obtained by the SA are compared to their corresponding optimal values which are reported under the column labeled as "M\&K" in Table 5.

The results reported in Tables 4 and 5 reveal that the proposed SA algorithm is able to obtain the optimal solutions for all the instances of the TR data set. From a solution time perspective, it is shown that the SA solves the problem instances for the TR data set in quite short CPU times. Another important observation from the these tables is that as the number of hubs opened by the leader $(p)$ increases, the follower fails to capture much of the market share even if $r>p$. One possible reason for this observation can be the fact that as $p$ increases, the leader selects more of the critical locations for opening hubs and reduces its cost. In addition, since the customers choose the leader's service for an equal cost offered by the leader and the follower, the leader's market share stays higher than that of the follower.

Table 6 shows the results for solving the multiple allocation $(r \mid p)$ hub-centroid problem for the CAB data set. To evaluate the performance of the proposed SA algorithm, the best solutions obtained by SA are compared to those of enumeration based algorithm presented in Mahmutogullari and Kara (2016) as it is not practical to solve the proposed bilevel model using CPLEX. 
Table 5

Results for multiple allocation $\left(r \mid X_{p}\right)$ hub-medianoid problem with the TR data set $(r, p \geq 6)$.

\begin{tabular}{|c|c|c|c|c|c|c|c|c|c|c|c|c|c|c|}
\hline \multicolumn{5}{|c|}{$\alpha=0.6$} & \multicolumn{5}{|c|}{$\alpha=0.8$} & \multicolumn{5}{|c|}{$\alpha=0.9$} \\
\hline \multirow[t]{2}{*}{$p$} & \multirow[t]{2}{*}{$r$} & \multirow{2}{*}{$\begin{array}{l}\text { M\&K } \\
\text { Follower's } \\
\text { capture }\end{array}$} & \multicolumn{2}{|l|}{ SA } & \multirow[t]{2}{*}{$p$} & \multirow[t]{2}{*}{$r$} & \multirow{2}{*}{$\begin{array}{l}\text { M\&K } \\
\text { Follower's } \\
\text { capture }\end{array}$} & \multicolumn{2}{|l|}{ SA } & \multirow[t]{2}{*}{$p$} & \multirow[t]{2}{*}{$r$} & \multirow{2}{*}{$\begin{array}{l}\text { M\&K } \\
\text { Follower's } \\
\text { capture }\end{array}$} & \multicolumn{2}{|l|}{ SA } \\
\hline & & & $\begin{array}{l}\text { Follower's } \\
\text { capture }\end{array}$ & CPU (s) & & & & $\begin{array}{l}\text { Follower's } \\
\text { capture }\end{array}$ & CPU (s) & & & & $\begin{array}{l}\text { Follower's } \\
\text { capture }\end{array}$ & CPU (s) \\
\hline \multirow[t]{5}{*}{6} & 6 & $39.31 \%$ & $39.31 \%$ & 2.25 & \multirow[t]{5}{*}{6} & 6 & $37.97 \%$ & $37.97 \%$ & 2.88 & \multirow[t]{5}{*}{6} & 6 & $40.86 \%$ & $40.86 \%$ & 4.83 \\
\hline & 8 & $49.19 \%$ & $49.19 \%$ & 4.31 & & 8 & $48.24 \%$ & $48.24 \%$ & 4.19 & & 8 & $49.44 \%$ & $49.44 \%$ & 7.39 \\
\hline & 10 & $56.94 \%$ & $56.94 \%$ & 5.55 & & 10 & $55.70 \%$ & $55.70 \%$ & 5.47 & & 10 & $56.06 \%$ & $56.04 \%$ & 9.80 \\
\hline & 12 & $64.02 \%$ & $64.02 \%$ & 6.98 & & 12 & $61.84 \%$ & $61.84 \%$ & 7.22 & & 12 & $61.54 \%$ & $61.54 \%$ & 13.38 \\
\hline & 14 & $68.91 \%$ & $68.91 \%$ & 8.27 & & 14 & $66.97 \%$ & $66.97 \%$ & 9.18 & & 14 & $66.45 \%$ & $66.45 \%$ & 16.92 \\
\hline \multirow[t]{5}{*}{8} & 6 & $28.58 \%$ & $28.58 \%$ & 4.25 & \multirow[t]{5}{*}{8} & 6 & $29.37 \%$ & $29.37 \%$ & 4.23 & \multirow[t]{5}{*}{8} & 6 & $31.11 \%$ & $31.10 \%$ & 7.34 \\
\hline & 8 & $37.09 \%$ & $37.09 \%$ & 5.30 & & 8 & $37.08 \%$ & $37.08 \%$ & 5.27 & & 8 & $38.69 \%$ & $38.69 \%$ & 9.17 \\
\hline & 10 & $44.37 \%$ & $44.37 \%$ & 9.22 & & 10 & $44.35 \%$ & $44.35 \%$ & 9.91 & & 10 & $44.83 \%$ & $44.83 \%$ & 12.57 \\
\hline & 12 & $51.77 \%$ & $51.77 \%$ & 12.29 & & 12 & $50.71 \%$ & $50.71 \%$ & 12.15 & & 12 & $50.49 \%$ & $50.49 \%$ & 15.69 \\
\hline & 14 & $57.97 \%$ & $57.97 \%$ & 15.40 & & 14 & $56.33 \%$ & $56.33 \%$ & 13.98 & & 14 & $55.77 \%$ & $55.77 \%$ & 19.14 \\
\hline \multirow[t]{5}{*}{10} & 6 & $19.91 \%$ & $19.91 \%$ & 8.53 & \multirow[t]{5}{*}{10} & 6 & $20.12 \%$ & $20.12 \%$ & 8.25 & \multirow[t]{5}{*}{10} & 6 & $20.74 \%$ & $20.74 \%$ & 10.01 \\
\hline & 8 & $27.13 \%$ & $27.13 \%$ & 9.87 & & 8 & $27.03 \%$ & $27.03 \%$ & 9.71 & & 8 & $27.77 \%$ & $27.77 \%$ & 11.12 \\
\hline & 10 & $34.10 \%$ & $34.10 \%$ & 12.20 & & 10 & $33.84 \%$ & $33.84 \%$ & 12.80 & & 10 & $33.86 \%$ & $33.86 \%$ & 14.82 \\
\hline & 12 & $40.48 \%$ & $40.48 \%$ & 13.98 & & 12 & $40.74 \%$ & $40.74 \%$ & 17.69 & & 12 & $39.89 \%$ & $39.89 \%$ & 17.50 \\
\hline & 14 & $45.73 \%$ & $45.73 \%$ & 19.92 & & 14 & $46.84 \%$ & $46.84 \%$ & 21.19 & & 14 & $44.90 \%$ & $44.90 \%$ & 21.35 \\
\hline \multirow[t]{5}{*}{12} & 6 & $15.83 \%$ & $15.83 \%$ & 11.08 & \multirow[t]{5}{*}{12} & 6 & $16.93 \%$ & $16.93 \%$ & 12.03 & \multirow[t]{5}{*}{12} & 6 & $18.45 \%$ & $18.45 \%$ & 11.90 \\
\hline & 8 & $21.79 \%$ & $21.79 \%$ & 13.15 & & 8 & $23.41 \%$ & $23.41 \%$ & 13.54 & & 8 & $24.59 \%$ & $24.59 \%$ & 13.58 \\
\hline & 10 & $27.06 \%$ & $27.06 \%$ & 17.43 & & 10 & $28.62 \%$ & $28.62 \%$ & 17.40 & & 10 & $29.08 \%$ & $29.08 \%$ & 17.79 \\
\hline & 12 & $31.37 \%$ & $31.37 \%$ & 20.77 & & 12 & $32.81 \%$ & $32.81 \%$ & 20.56 & & 12 & $32.98 \%$ & $32.98 \%$ & 20.74 \\
\hline & 14 & $35.48 \%$ & $35.48 \%$ & 23.85 & & 14 & $35.85 \%$ & $35.93 \%$ & 23.75 & & 14 & $36.18 \%$ & $36.18 \%$ & 24.15 \\
\hline 14 & 6 & $13.04 \%$ & $13.04 \%$ & 14.70 & 14 & 6 & $13.02 \%$ & $13.02 \%$ & 14.68 & 14 & 6 & $13.66 \%$ & $13.66 \%$ & 15.01 \\
\hline & 8 & $17.87 \%$ & $17.87 \%$ & 16.58 & & 8 & $18.57 \%$ & $18.57 \%$ & 16.91 & & 8 & $18.81 \%$ & $18.81 \%$ & 16.43 \\
\hline & 10 & $22.25 \%$ & $22.25 \%$ & 20.95 & & 10 & $22.52 \%$ & $22.52 \%$ & 23.02 & & 10 & $22.50 \%$ & $22.50 \%$ & 21.59 \\
\hline & 12 & $26.00 \%$ & $26.00 \%$ & 25.26 & & 12 & $25.20 \%$ & $25.20 \%$ & 25.09 & & 12 & $25.60 \%$ & $25.60 \%$ & 24.68 \\
\hline & 14 & $28.42 \%$ & $28.42 \%$ & 27.31 & & 14 & $27.40 \%$ & $27.46 \%$ & 27.60 & & 14 & $28.18 \%$ & $28.18 \%$ & 27.24 \\
\hline Ave & & $36.18 \%$ & $36.18 \%$ & 13.17 & Ave & & $36.06 \%$ & $36.06 \%$ & 13.54 & Ave & & $36.50 \%$ & $36.50 \%$ & 15.36 \\
\hline
\end{tabular}

Table 6

Results for multiple allocation $(r \mid p)$ hub-centroid problem with the CAB data set.

\begin{tabular}{|c|c|c|c|c|c|c|c|c|c|}
\hline \multicolumn{5}{|c|}{$\alpha=0.6$} & \multicolumn{5}{|c|}{$\alpha=0.8$} \\
\hline \multirow[t]{2}{*}{$p$} & \multirow[t]{2}{*}{$r$} & \multirow{2}{*}{$\begin{array}{l}\text { M\&K } \\
\text { Follower's capture }\end{array}$} & \multicolumn{2}{|l|}{ SA } & \multirow[t]{2}{*}{$p$} & \multirow[t]{2}{*}{$r$} & \multirow{2}{*}{$\begin{array}{l}\text { M\&K } \\
\text { Follower's capture }\end{array}$} & \multicolumn{2}{|l|}{ SA } \\
\hline & & & Follower's capture & $\mathrm{CPU}(\mathrm{s})$ & & & & Follower's capture & $\mathrm{CPU}(\mathrm{s})$ \\
\hline \multirow[t]{4}{*}{2} & 2 & $46.14 \%$ & $46.14 \%$ & 9.50 & \multirow[t]{4}{*}{2} & 2 & $43.68 \%$ & $43.68 \%$ & 10.06 \\
\hline & 3 & $64.37 \%$ & $64.37 \%$ & 17.87 & & 3 & $59.59 \%$ & $59.59 \%$ & 17.41 \\
\hline & 4 & $74.75 \%$ & $74.75 \%$ & 38.37 & & 4 & $70.75 \%$ & $70.75 \%$ & 38.61 \\
\hline & 5 & $83.52 \%$ & $83.52 \%$ & 83.61 & & 5 & $78.74 \%$ & $78.74 \%$ & 83.37 \\
\hline \multirow[t]{4}{*}{3} & 2 & $30.39 \%$ & $30.39 \%$ & 14.40 & \multirow[t]{4}{*}{3} & 2 & $29.18 \%$ & $29.18 \%$ & 14.35 \\
\hline & 3 & $45.13 \%$ & $45.13 \%$ & 23.93 & & 3 & $42.87 \%$ & $42.87 \%$ & 23.23 \\
\hline & 4 & $53.69 \%$ & $53.69 \%$ & 48.84 & & 4 & $52.83 \%$ & $52.83 \%$ & 47.18 \\
\hline & 5 & $62.02 \%$ & $62.02 \%$ & 98.52 & & 5 & $60.14 \%$ & $60.14 \%$ & 97.81 \\
\hline \multirow[t]{4}{*}{4} & 2 & $17.91 \%$ & $17.91 \%$ & 22.62 & \multirow[t]{4}{*}{4} & 2 & $21.06 \%$ & $21.06 \%$ & 26.71 \\
\hline & 3 & $28.39 \%$ & $28.39 \%$ & 37.76 & & 3 & $30.70 \%$ & $30.70 \%$ & 36.96 \\
\hline & 4 & $37.73 \%$ & $37.73 \%$ & 59.73 & & 4 & $38.39 \%$ & $38.39 \%$ & 56.94 \\
\hline & 5 & $46.18 \%$ & $46.18 \%$ & 121.16 & & 5 & $45.24 \%$ & $45.24 \%$ & 146.78 \\
\hline \multirow[t]{4}{*}{5} & 2 & $14.30 \%$ & $14.30 \%$ & 53.77 & \multirow[t]{4}{*}{5} & 2 & $15.30 \%$ & $15.30 \%$ & 58.7 \\
\hline & 3 & $23.73 \%$ & $23.73 \%$ & 132.68 & & 3 & $23.24 \%$ & $23.24 \%$ & 150.11 \\
\hline & 4 & $31.91 \%$ & $31.91 \%$ & 177.56 & & 4 & $31.78 \%$ & $31.78 \%$ & 180.95 \\
\hline & 5 & $39.58 \%$ & $39.58 \%$ & 226.55 & & 5 & $38.57 \%$ & $38.57 \%$ & 232.93 \\
\hline \multicolumn{2}{|c|}{ Average } & $43.73 \%$ & $43.73 \%$ & 72.92 & \multicolumn{2}{|c|}{ Average } & $42.63 \%$ & $42.63 \%$ & 76.38 \\
\hline
\end{tabular}

The results for solving the multiple allocation $(r \mid p)$ hub-centroid problem for the TR data set with $r, p \leq 5$ are presented in Table 7.

As can be seen from Tables 6 and 7, the proposed SA algorithm for the multiple allocation $(r \mid p)$ hub-centroid problem has found the optimal solution in all of the test instances. Note that the solution times for the $(r \mid p)$ hub-centroid problem are higher than the corresponding solution times for the $\left(r \mid X_{p}\right)$ hub-medianoid problem. This is due to the bilevel nature of the former problem which requires our proposed SA to solve the follower's problem from scratch whenever a new solution for the leader is found. However, the solution times for the bilevel problem are still quite short for a strategic planning problem such as locating facilities in a competitive environment.

It should be mentioned that the leader's market share has increased as he/she has decided based on $(r \mid p)$ hub-centroid prob- lem. In other words, taking into account the competition, the leader locates its hubs in such a way that the follower can capture as low flow as possible when he/she enters the market. For example, in the CAB data set with $\alpha=0.8$, in case the leader and follower both open 2 hubs, i.e. $p=r=2$, the value of captured market share by the follower when the leader ignores the competition and decides on the location of its hubs solely based on cost factors is around 66\%, whereas the corresponding capture by the follower drops to $44 \%$ as the leader acts in anticipation of an upcoming competition.

Table 8 shows the results obtained by solving the multiple allocation $(r \mid p)$ hub-centroid problem with the TR data set for large values of $r$ and $p(r, p \geq 6)$. The problem for large values of $r$ and $p$ has not been solved by the enumeration algorithm in Mahmutogullari and Kara (2016) due to memory requirements and 
Table 7

Results for multiple allocation $(r \mid p)$ hub-centroid problem with the TR data set $(r, p \leq 5)$.

\begin{tabular}{|c|c|c|c|c|c|c|c|c|c|c|c|c|c|c|}
\hline \multicolumn{5}{|c|}{$\alpha=0.6$} & \multicolumn{5}{|c|}{$\alpha=0.8$} & \multicolumn{5}{|c|}{$\alpha=0.9$} \\
\hline \multirow[t]{2}{*}{$p$} & \multirow[t]{2}{*}{$r$} & \multirow{2}{*}{$\begin{array}{l}\text { M\&K } \\
\text { Follower's } \\
\text { capture }\end{array}$} & \multicolumn{2}{|l|}{ SA } & \multirow[t]{2}{*}{$p$} & \multirow[t]{2}{*}{$r$} & \multirow{2}{*}{$\begin{array}{l}\text { M\&K } \\
\text { Follower's } \\
\text { capture }\end{array}$} & \multicolumn{2}{|l|}{ SA } & \multirow[t]{2}{*}{$p$} & \multirow[t]{2}{*}{$r$} & \multirow{2}{*}{$\begin{array}{l}\text { M\&K } \\
\text { Follower's } \\
\text { capture }\end{array}$} & \multicolumn{2}{|l|}{ SA } \\
\hline & & & $\begin{array}{l}\text { Follower's } \\
\text { capture }\end{array}$ & CPU (s) & & & & $\begin{array}{l}\text { Follower's } \\
\text { capture }\end{array}$ & CPU (s) & & & & $\begin{array}{l}\text { Follower's } \\
\text { capture }\end{array}$ & CPU (s) \\
\hline \multirow[t]{4}{*}{2} & 2 & $49.44 \%$ & $49.44 \%$ & 74.59 & \multirow[t]{4}{*}{2} & 2 & $46.84 \%$ & $46.84 \%$ & 75.43 & \multirow[t]{4}{*}{2} & 2 & $44.12 \%$ & $44.12 \%$ & 72.63 \\
\hline & 3 & $64.65 \%$ & $64.65 \%$ & 112.09 & & 3 & $60.05 \%$ & $60.05 \%$ & 108.23 & & 3 & $58.74 \%$ & $58.74 \%$ & 108.91 \\
\hline & 4 & $74.97 \%$ & $74.97 \%$ & 148.93 & & 4 & $70.03 \%$ & $70.03 \%$ & 155.33 & & 4 & $67.98 \%$ & $67.98 \%$ & 155.19 \\
\hline & 5 & $84.72 \%$ & $84.72 \%$ & 210.54 & & 5 & $77.97 \%$ & $77.97 \%$ & 218.44 & & 5 & $75.45 \%$ & $75.45 \%$ & 210.10 \\
\hline \multirow[t]{4}{*}{3} & 2 & $30.49 \%$ & $30.49 \%$ & 108.30 & \multirow[t]{4}{*}{3} & 2 & $30.68 \%$ & $30.68 \%$ & 108.52 & \multirow[t]{4}{*}{3} & 2 & $30.35 \%$ & $30.35 \%$ & 107.52 \\
\hline & 3 & $40.82 \%$ & $40.82 \%$ & 145.17 & & 3 & $40.81 \%$ & $40.81 \%$ & 146.58 & & 3 & $39.90 \%$ & $39.90 \%$ & 140.42 \\
\hline & 4 & $56.18 \%$ & $56.18 \%$ & 185.27 & & 4 & $51.43 \%$ & $51.43 \%$ & 184.20 & & 4 & $50.03 \%$ & $50.03 \%$ & 191.55 \\
\hline & 5 & $65.58 \%$ & $65.58 \%$ & 251.48 & & 5 & $60.66 \%$ & $60.66 \%$ & 250.41 & & 5 & $58.18 \%$ & $58.18 \%$ & 250.18 \\
\hline \multirow[t]{4}{*}{4} & 2 & $20.07 \%$ & $20.07 \%$ & 149.82 & \multirow[t]{4}{*}{4} & 2 & $20.33 \%$ & $20.33 \%$ & 160.12 & \multirow[t]{4}{*}{4} & 2 & $20.38 \%$ & $20.38 \%$ & 154.09 \\
\hline & 3 & $30.57 \%$ & $30.57 \%$ & 194.42 & & 3 & $30.19 \%$ & $30.19 \%$ & 198.59 & & 3 & $29.55 \%$ & $29.55 \%$ & 198.44 \\
\hline & 4 & $42.15 \%$ & $42.15 \%$ & 242.14 & & 4 & $39.41 \%$ & $39.41 \%$ & 233.58 & & 4 & $38.11 \%$ & $38.11 \%$ & 231.55 \\
\hline & 5 & $51.89 \%$ & $51.89 \%$ & 322.75 & & 5 & $48.57 \%$ & $48.57 \%$ & 302.39 & & 5 & $46.83 \%$ & $46.83 \%$ & 300.12 \\
\hline \multirow[t]{4}{*}{5} & 2 & $14.32 \%$ & $14.32 \%$ & 210.27 & \multirow[t]{4}{*}{5} & 2 & $14.82 \%$ & $14.82 \%$ & 220.39 & \multirow[t]{4}{*}{5} & 2 & $14.27 \%$ & $14.27 \%$ & 212.61 \\
\hline & 3 & $23.61 \%$ & $23.61 \%$ & 275.00 & & 3 & $22.12 \%$ & $22.12 \%$ & 294.22 & & 3 & $22.87 \%$ & $22.87 \%$ & 317.82 \\
\hline & 4 & $32.34 \%$ & $32.34 \%$ & 306.76 & & 4 & $29.28 \%$ & $29.28 \%$ & 314.83 & & 4 & $31.76 \%$ & $31.76 \%$ & 401.35 \\
\hline & 5 & $40.05 \%$ & $40.05 \%$ & 358.91 & & 5 & $37.44 \%$ & $37.44 \%$ & 471.31 & & 5 & $38.91 \%$ & $38.91 \%$ & 485.00 \\
\hline \multicolumn{2}{|c|}{ Average } & $45.11 \%$ & $45.11 \%$ & 206.02 & \multicolumn{2}{|c|}{ Average } & $42.53 \%$ & $42.53 \%$ & 215.16 & \multicolumn{2}{|c|}{ Average } & $41.71 \%$ & $41.71 \%$ & 221.09 \\
\hline
\end{tabular}

Table 8

Results for multiple allocation $(r \mid p)$ hub-centroid problem with the TR data set $(r, p \geq 6)$.

\begin{tabular}{|c|c|c|c|c|c|c|c|c|c|c|c|}
\hline \multicolumn{4}{|c|}{$\alpha=0.6$} & \multicolumn{4}{|c|}{$\alpha=0.8$} & \multicolumn{4}{|c|}{$\alpha=0.9$} \\
\hline \multirow[t]{2}{*}{$p$} & \multirow[t]{2}{*}{$r$} & \multicolumn{2}{|l|}{ SA } & \multirow[t]{2}{*}{$p$} & \multirow[t]{2}{*}{$r$} & \multicolumn{2}{|l|}{ SA } & \multirow[t]{2}{*}{$p$} & \multirow[t]{2}{*}{$r$} & \multicolumn{2}{|l|}{ SA } \\
\hline & & Follower's capture & $\mathrm{CPU}(\mathrm{s})$ & & & Follower's capture & CPU (s) & & & Follower's capture & $\mathrm{CPU}(\mathrm{s})$ \\
\hline \multirow[t]{5}{*}{6} & 6 & $39.29 \%$ & 2115.64 & \multirow[t]{5}{*}{6} & 6 & $37.15 \%$ & 2111.09 & \multirow[t]{5}{*}{6} & 6 & $36.77 \%$ & 2134.08 \\
\hline & 8 & $49.12 \%$ & 2359.87 & & 8 & $47.70 \%$ & 2287.74 & & 8 & $47.27 \%$ & 2490.12 \\
\hline & 10 & $56.94 \%$ & 2871.65 & & 10 & $55.70 \%$ & 2901.14 & & 10 & $54.96 \%$ & 2752.40 \\
\hline & 12 & $64.02 \%$ & 3094.27 & & 12 & $61.84 \%$ & 2931.95 & & 12 & $60.96 \%$ & 3093.01 \\
\hline & 14 & $68.85 \%$ & 3300.69 & & 14 & $66.97 \%$ & 2219.11 & & 14 & $65.67 \%$ & 3318.89 \\
\hline \multirow[t]{5}{*}{8} & 6 & $28.45 \%$ & 2495.08 & \multirow[t]{5}{*}{8} & 6 & $27.65 \%$ & 2339.75 & \multirow[t]{5}{*}{8} & 6 & $27.62 \%$ & 2499.02 \\
\hline & 8 & $36.34 \%$ & 2950.11 & & 8 & $35.62 \%$ & 3008.21 & & 8 & $36.11 \%$ & 2876.44 \\
\hline & 10 & $43.44 \%$ & 3221.27 & & 10 & $42.72 \%$ & 3225.41 & & 10 & $42.52 \%$ & 3339.01 \\
\hline & 12 & $50.15 \%$ & 3950.67 & & 12 & $49.63 \%$ & 3718.72 & & 12 & $48.37 \%$ & 3718.72 \\
\hline & 14 & $54.98 \%$ & 4391.55 & & 14 & $53.74 \%$ & 4182.51 & & 14 & $53.91 \%$ & 4391.65 \\
\hline \multirow[t]{5}{*}{10} & 6 & $19.91 \%$ & 2683.80 & \multirow[t]{5}{*}{10} & 6 & $20.12 \%$ & 2699.10 & \multirow[t]{5}{*}{10} & 6 & $20.74 \%$ & 2591.03 \\
\hline & 8 & $27.13 \%$ & 2974.01 & & 8 & $27.03 \%$ & 2940.69 & & 8 & $27.77 \%$ & 3004.10 \\
\hline & 10 & $34.10 \%$ & 3409.31 & & 10 & $33.75 \%$ & 3519.09 & & 10 & $33.86 \%$ & 3581.93 \\
\hline & 12 & $39.14 \%$ & 3954.94 & & 12 & $40.58 \%$ & 4021.39 & & 12 & $39.80 \%$ & 4056.88 \\
\hline & 14 & $43.56 \%$ & 4487.39 & & 14 & $43.43 \%$ & 4527.71 & & 14 & $44.07 \%$ & 4681.53 \\
\hline \multirow[t]{5}{*}{12} & 6 & $14.83 \%$ & 2720.55 & \multirow[t]{5}{*}{12} & 6 & $15.75 \%$ & 2751.48 & \multirow[t]{5}{*}{12} & 6 & $16.18 \%$ & 2719.97 \\
\hline & 8 & $21.21 \%$ & 3363.98 & & 8 & $21.86 \%$ & 3495.30 & & 8 & $22.36 \%$ & 3387.13 \\
\hline & 10 & $27.00 \%$ & 3890.27 & & 10 & $28.10 \%$ & 3786.59 & & 10 & $27.68 \%$ & 3810.60 \\
\hline & 12 & $31.22 \%$ & 4319.92 & & 12 & $32.35 \%$ & 4385.35 & & 12 & $31.99 \%$ & 4250.71 \\
\hline & 14 & $35.16 \%$ & 4974.31 & & 14 & $35.28 \%$ & 5009.50 & & 14 & $35.05 \%$ & 4971.48 \\
\hline \multirow[t]{5}{*}{14} & 6 & $12.34 \%$ & 3511.90 & \multirow[t]{5}{*}{14} & 6 & $11.95 \%$ & 3419.82 & \multirow[t]{5}{*}{14} & 6 & $12.31 \%$ & 3445.77 \\
\hline & 8 & $17.40 \%$ & 3901.25 & & 8 & $17.36 \%$ & 3975.01 & & 8 & $17.13 \%$ & 3959.24 \\
\hline & 10 & $21.79 \%$ & 4458.14 & & 10 & $22.28 \%$ & 4590.25 & & 10 & $21.57 \%$ & 4611.38 \\
\hline & 12 & $25.13 \%$ & 4975.68 & & 12 & $25.15 \%$ & 5001.81 & & 12 & $24.47 \%$ & 4988.17 \\
\hline & 14 & $27.15 \%$ & 5721.24 & & 14 & $27.24 \%$ & 5789.61 & & 14 & $26.89 \%$ & 5608.55 \\
\hline \multicolumn{2}{|c|}{ Average } & $35.55 \%$ & 3603.90 & \multicolumn{2}{|c|}{ Average } & $35.24 \%$ & 3553.53 & Ave & & $35.04 \%$ & 3611.27 \\
\hline
\end{tabular}

long CPU times. For this reason, we present only the results obtained by the proposed SA algorithm in this table.

Note from Table 8 that for larger values of the installed hubs $(r, p \geq 6)$, the follower's capture is, in general, less than the corresponding value when the number of installed hubs are smaller $(r$, $p \leq 5$ ). It can also be seen that all the instances of the problem with large values of $r, p$ are solved in less than an hour on average. Since these instances have not already been solved using exact solution algorithms or CPLEX, the obtained results can be considered as an indication of the usefulness and high efficiency of the proposed SA algorithm.

\subsection{Results for the single allocation case}

Results obtained by solving the single allocation $\left(r \mid X_{p}\right)$ hubmedianoid problem using the proposed SA algorithm as well as
CPLEX based on the proposed mathematical models with the CAB data set are presented in Table 9. It is assumed that the leader has already located its hubs based on the uncapacitated single allocation $p$-hub median problem (USApHMP). Similar to the case of multiple allocation, to reduce the size of our model, the constraints (24)-(25) are imposed for only $i<j$ and the objective (20) is modified as $\sum_{i} \sum_{j \mid j>i}\left(w_{i j}+w_{j i}\right) z_{i j}$ in our computational studies.

It can be seen from Table 9 that the proposed SA algorithm solves all the instances of the single allocation $\left(r \mid X_{p}\right)$ hubmedianoid problem to optimality in less than a second. It should also be noted that CPLEX solves the single allocation medianoid instances within shorter times than the corresponding multiple allocation instances. This shows the model proposed for the single allocation problem which is based on the efficient formulation presented by Peker and Kara (2015) is more efficient than the multiple 
Table 9

Results for single allocation $\left(r \mid X_{p}\right)$ hub-medianoid problem with the CAB data set.

\begin{tabular}{|c|c|c|c|c|c|c|c|c|c|c|c|}
\hline \multicolumn{6}{|c|}{$\alpha=0.6$} & \multicolumn{6}{|c|}{$\alpha=0.8$} \\
\hline \multirow[t]{2}{*}{$p$} & \multirow[t]{2}{*}{$r$} & \multicolumn{2}{|l|}{ CPLEX } & \multicolumn{2}{|l|}{ SA } & \multirow[t]{2}{*}{$p$} & \multirow[t]{2}{*}{$r$} & \multicolumn{2}{|l|}{ CPLEX } & \multicolumn{2}{|l|}{ SA } \\
\hline & & Follower's capture & CPU (s) & Follower's capture & $\mathrm{CPU}(\mathrm{s})$ & & & Follower's capture & $\mathrm{CPU}(\mathrm{s})$ & Follower's capture & CPU (s) \\
\hline \multirow[t]{4}{*}{2} & 2 & $62.11 \%$ & 6.33 & $62.11 \%$ & 0.33 & \multirow[t]{4}{*}{2} & 2 & $61.25 \%$ & 2.10 & $61.25 \%$ & 0.37 \\
\hline & 3 & $78.46 \%$ & 3.35 & $78.46 \%$ & 0.44 & & 3 & $70.41 \%$ & 3.13 & $70.41 \%$ & 0.48 \\
\hline & 4 & $88.90 \%$ & 1.63 & $88.90 \%$ & 0.53 & & 4 & $80.85 \%$ & 2.59 & $80.85 \%$ & 0.54 \\
\hline & 5 & $93.49 \%$ & 1.49 & $93.49 \%$ & 0.61 & & 5 & $89.24 \%$ & 1.49 & $89.24 \%$ & 0.62 \\
\hline \multirow[t]{4}{*}{3} & 2 & $42.56 \%$ & 3.27 & $42.56 \%$ & 0.38 & \multirow[t]{4}{*}{3} & 2 & $46.27 \%$ & 2.65 & $46.27 \%$ & 0.34 \\
\hline & 3 & $59.00 \%$ & 6.62 & $59.00 \%$ & 0.45 & & 3 & $60.86 \%$ & 4.70 & $60.86 \%$ & 0.43 \\
\hline & 4 & $71.32 \%$ & 2.46 & $71.32 \%$ & 0.52 & & 4 & $73.04 \%$ & 1.15 & $73.04 \%$ & 0.55 \\
\hline & 5 & $83.41 \%$ & 0.74 & $83.41 \%$ & 0.61 & & 5 & $82.99 \%$ & 0.65 & $82.99 \%$ & 0.62 \\
\hline \multirow[t]{4}{*}{4} & 2 & $28.53 \%$ & 3.84 & $28.53 \%$ & 0.46 & \multirow[t]{4}{*}{4} & 2 & $41.42 \%$ & 1.51 & $41.42 \%$ & 0.35 \\
\hline & 3 & $36.98 \%$ & 10.51 & $36.98 \%$ & 0.47 & & 3 & $49.04 \%$ & 2.79 & $49.04 \%$ & 0.40 \\
\hline & 4 & $44.39 \%$ & 9.20 & $44.39 \%$ & 0.49 & & 4 & $57.23 \%$ & 2.52 & $57.23 \%$ & 0.51 \\
\hline & 5 & $51.73 \%$ & 8.07 & $51.73 \%$ & 0.63 & & 5 & $64.70 \%$ & 2.23 & $64.70 \%$ & 0.62 \\
\hline \multirow[t]{4}{*}{5} & 2 & $26.16 \%$ & 3.82 & $26.16 \%$ & 0.36 & \multirow[t]{4}{*}{5} & 2 & $39.12 \%$ & 1.17 & $39.12 \%$ & 0.41 \\
\hline & 3 & $33.62 \%$ & 6.04 & $33.62 \%$ & 0.47 & & 3 & $46.92 \%$ & 1.71 & $46.92 \%$ & 0.42 \\
\hline & 4 & $39.43 \%$ & 6.08 & $39.43 \%$ & 0.51 & & 4 & $53.35 \%$ & 2.15 & $53.35 \%$ & 0.58 \\
\hline & 5 & $45.01 \%$ & 6.94 & $45.01 \%$ & 0.65 & & 5 & $59.52 \%$ & 2.21 & $59.52 \%$ & 0.67 \\
\hline \multicolumn{2}{|c|}{ Average } & $55.32 \%$ & 5.02 & $55.32 \%$ & 0.49 & \multicolumn{2}{|c|}{ Average } & $61.01 \%$ & 2.17 & $61.01 \%$ & 0.49 \\
\hline
\end{tabular}

Table 10

Results for single allocation $\left(r \mid X_{p}\right)$ hub-medianoid problem with the TR data set $(r, p \leq 5)$.

\begin{tabular}{|c|c|c|c|c|c|c|c|c|c|c|c|c|c|c|c|c|c|}
\hline \multicolumn{6}{|c|}{$\alpha=0.6$} & \multicolumn{6}{|c|}{$\alpha=0.8$} & \multicolumn{6}{|c|}{$\alpha=0.9$} \\
\hline \multirow[t]{2}{*}{$p$} & \multirow[t]{2}{*}{$r$} & \multicolumn{2}{|l|}{ CPLEX } & \multicolumn{2}{|l|}{ SA } & \multirow[t]{2}{*}{$p$} & \multirow[t]{2}{*}{$r$} & \multicolumn{2}{|l|}{ CPLEX } & \multicolumn{2}{|l|}{ SA } & \multirow[t]{2}{*}{$p$} & \multirow[t]{2}{*}{$r$} & \multicolumn{2}{|l|}{ CPLEX } & \multicolumn{2}{|l|}{ SA } \\
\hline & & $\begin{array}{l}\text { Follower's } \\
\text { capture }\end{array}$ & CPU (s) & $\begin{array}{l}\text { Follower's } \\
\text { capture }\end{array}$ & $\begin{array}{l}\text { CPU } \\
(s)\end{array}$ & & & $\begin{array}{l}\text { Follower's } \\
\text { capture }\end{array}$ & CPU (s) & $\begin{array}{l}\text { Follower's } \\
\text { capture }\end{array}$ & CPU (s) & & & $\begin{array}{l}\text { Follower's } \\
\text { capture }\end{array}$ & CPU (s) & $\begin{array}{l}\text { Follower's } \\
\text { capture }\end{array}$ & CPU (s) \\
\hline \multirow[t]{4}{*}{2} & 2 & $51.07 \%$ & 216.02 & $51.07 \%$ & 7.15 & \multirow[t]{4}{*}{2} & 2 & $50.52 \%$ & 145.68 & $50.52 \%$ & 8.45 & \multirow[t]{4}{*}{2} & 2 & $49.27 \%$ & 104.16 & $49.27 \%$ & 8.66 \\
\hline & 3 & $63.56 \%$ & 647.37 & $63.56 \%$ & 11.98 & & 3 & $62.67 \%$ & 228.64 & $62.67 \%$ & 13.58 & & 3 & $63.14 \%$ & 135.11 & $63.14 \%$ & 13.32 \\
\hline & 4 & $73.89 \%$ & 628.58 & $73.89 \%$ & 14.34 & & 4 & $75.74 \%$ & 118.99 & $75.74 \%$ & 15.44 & & 4 & $73.41 \%$ & 153.20 & $73.41 \%$ & 16.01 \\
\hline & 5 & $81.23 \%$ & 683.59 & $81.23 \%$ & 16.85 & & 5 & $83.42 \%$ & 35.17 & $83.42 \%$ & 17.05 & & 5 & $79.91 \%$ & 49.15 & $79.91 \%$ & 17.23 \\
\hline \multirow[t]{4}{*}{3} & 2 & $39.37 \%$ & 296.05 & $39.37 \%$ & 8.47 & \multirow[t]{4}{*}{3} & 2 & $54.38 \%$ & 96.18 & $54.38 \%$ & 9.00 & \multirow[t]{4}{*}{3} & 2 & $59.09 \%$ & 42.28 & $59.09 \%$ & 8.54 \\
\hline & 3 & $48.81 \%$ & 568.05 & $48.81 \%$ & 13.01 & & 3 & $62.41 \%$ & 186.37 & $62.41 \%$ & 13.51 & & 3 & $63.48 \%$ & 62.51 & $63.48 \%$ & 13.51 \\
\hline & 4 & $58.63 \%$ & 888.42 & $58.63 \%$ & 14.64 & & 4 & $68.43 \%$ & 141.08 & $68.43 \%$ & 15.90 & & 4 & $68.88 \%$ & 80.31 & $68.88 \%$ & 15.09 \\
\hline & 5 & $67.55 \%$ & 757.60 & $67.55 \%$ & 17.08 & & 5 & $74.73 \%$ & 129.01 & $74.73 \%$ & 17.66 & & 5 & $74.01 \%$ & 73.23 & $74.01 \%$ & 17.98 \\
\hline \multirow[t]{4}{*}{4} & 2 & $31.57 \%$ & 174.93 & $31.57 \%$ & 9.00 & \multirow[t]{4}{*}{4} & 2 & $34.20 \%$ & 122.62 & $34.20 \%$ & 8.49 & \multirow[t]{4}{*}{4} & 2 & $35.33 \%$ & 129.61 & $35.33 \%$ & 8.94 \\
\hline & 3 & $39.81 \%$ & 530.30 & $39.81 \%$ & 13.82 & & 3 & $38.63 \%$ & 385.19 & $38.63 \%$ & 13.98 & & 3 & $42.59 \%$ & 147.03 & $42.59 \%$ & 13.88 \\
\hline & 4 & $46.78 \%$ & 642.57 & $46.78 \%$ & 15.02 & & 4 & $46.61 \%$ & 312.51 & $46.61 \%$ & 16.15 & & 4 & $49.21 \%$ & 358.67 & $49.21 \%$ & 15.67 \\
\hline & 5 & $55.73 \%$ & 374.78 & $55.73 \%$ & 17.37 & & 5 & $53.83 \%$ & 197.53 & $53.83 \%$ & 18.42 & & 5 & $55.18 \%$ & 298.61 & $55.18 \%$ & 17.96 \\
\hline \multirow[t]{4}{*}{5} & 2 & $25.03 \%$ & 102.02 & $25.03 \%$ & 7.18 & \multirow[t]{4}{*}{5} & 2 & $29.90 \%$ & 95.22 & $29.90 \%$ & 8.07 & \multirow[t]{4}{*}{5} & 2 & $32.15 \%$ & 109.18 & $32.15 \%$ & 8.00 \\
\hline & 3 & $30.63 \%$ & 319.49 & $30.63 \%$ & 13.46 & & 3 & $34.46 \%$ & 248.49 & $34.46 \%$ & 14.22 & & 3 & $37.51 \%$ & 128.94 & $37.51 \%$ & 14.11 \\
\hline & 4 & $37.91 \%$ & 554.96 & $37.91 \%$ & 15.39 & & 4 & $38.87 \%$ & 407.86 & $38.87 \%$ & 15.94 & & 4 & $41.95 \%$ & 235.30 & $41.95 \%$ & 15.91 \\
\hline & 5 & $45.04 \%$ & 357.46 & $45.04 \%$ & 17.63 & & 5 & $45.14 \%$ & 416.34 & $45.14 \%$ & 18.12 & & 5 & $45.99 \%$ & 381.36 & $45.99 \%$ & 18.26 \\
\hline \multicolumn{2}{|c|}{ Average } & $49.78 \%$ & 483.89 & $49.78 \%$ & 13.27 & \multicolumn{2}{|c|}{ Average } & $53.37 \%$ & 204.18 & $53.37 \%$ & 13.99 & \multicolumn{2}{|c|}{ Average } & $54.44 \%$ & 155.54 & $54.44 \%$ & 13.94 \\
\hline
\end{tabular}

allocation model although the single allocation problem is more difficult in nature. Furthermore, it is observed that when the leader locates its hubs based on the $p$-hub median problem, the captured flow by the follower is higher in case of the single allocation network than that of the corresponding multiple allocation network.

Corresponding results for the TR data set with $p, r \leq 5$ and $p$, $r \geq 6$ are presented in Tables 10 and 11, respectively.

Observe from the Tables 10 and 11 that the proposed SA algorithm for the single allocation $\left(r \mid X_{p}\right)$ hub-medianoid problem is also able to obtain the optimal solutions for all the instances in the TR data set. Note that the proposed SA solves the problem instances for the TR data set in quite short CPU times. Furthermore, it can be seen that the CPU time taken by CPLEX to solve the instances with small values of $p$ and $r(r, p \leq 5)$ is, on average, longer that the solution times for instances with larger $p$ and $r$ values $(r, p \geq 6)$. Another interesting observation from these results is in connection to the ability of the follower in capturing the market share under the two allocation settings. Comparing the results for the single allocation medianoid problem with those of the multiple allocation problem, one can observe that the follower's captured flows in single allocation networks are generally higher than the corresponding multiple allocation networks. In other words, for the same values of the parameters $p, r$, and $\alpha$, the follower is able to gain a larger portion of the market in a single allocation network compared to a multiple allocation network when the leader locates its hubs based on the $p$-hub median problem for both the $\mathrm{CAB}$ and TR data sets.

Before testing the proposed SA algorithm for solving the single allocation $(r \mid p)$ hub-centroid problem on the main instances from the $C A B$ and TR data sets, we evaluate its performance by comparing to an adapted version of the enumeration algorithm from Mahmutogullari and Kara (2016). We coded the "smart$50 \%$ " version of the algorithm presented in Mahmutogullari and Kara (2016) and solved a number of small instances from the CAB data set. The experiments are conducted for first $n$ nodes of the CAB data set with $n \in\{10,15\}$, and $p, r \in\{2,3\}$. Table 12 summarizes the results of the computational study for these instances within a time limit of $10,800 \mathrm{~s}(3 \mathrm{~h})$.

Note from Table 12 that the enumeration algorithm solves the instances with $n=10$ to optimality in short computational times. However, the algorithm solves the instances with $n=15$ for only $p=r=2$. The solution times for these instances are substantially longer compared to the case of $n=10$. It can be seen that the enumeration algorithm cannot solve the problem for $n=15$ with 
Table 11

Results for single allocation $\left(r \mid X_{p}\right)$ hub-medianoid problem with the TR data set $(r, p \geq 6)$.

\begin{tabular}{|c|c|c|c|c|c|c|c|c|c|c|c|c|c|c|c|c|c|}
\hline \multicolumn{6}{|c|}{$\alpha=0.6$} & \multicolumn{6}{|c|}{$\alpha=0.8$} & \multicolumn{6}{|c|}{$\alpha=0.9$} \\
\hline \multirow[t]{2}{*}{$p$} & \multirow[t]{2}{*}{$r$} & \multicolumn{2}{|l|}{ CPLEX } & \multicolumn{2}{|l|}{ SA } & \multirow[t]{2}{*}{$\bar{p}$} & \multirow[t]{2}{*}{$r$} & \multicolumn{2}{|l|}{ CPLEX } & \multicolumn{2}{|l|}{ SA } & \multirow[t]{2}{*}{$\bar{p}$} & \multirow[t]{2}{*}{$r$} & \multicolumn{2}{|l|}{ CPLEX } & \multicolumn{2}{|l|}{ SA } \\
\hline & & $\begin{array}{l}\text { Follower's } \\
\text { capture }\end{array}$ & CPU (s) & $\begin{array}{l}\text { Follower's } \\
\text { capture }\end{array}$ & CPU (s) & & & $\begin{array}{l}\text { Follower's } \\
\text { capture }\end{array}$ & CPU (s) & $\begin{array}{l}\text { Follower's } \\
\text { capture }\end{array}$ & CPU (s) & & & $\begin{array}{l}\text { Follower's } \\
\text { capture }\end{array}$ & CPU (s) & $\begin{array}{l}\text { Follower's } \\
\text { capture }\end{array}$ & $\mathrm{CPU}(\mathrm{s})$ \\
\hline \multirow[t]{5}{*}{6} & 6 & $45.41 \%$ & 260.13 & $45.41 \%$ & 20.15 & \multirow[t]{5}{*}{6} & 6 & $46.13 \%$ & 471.67 & $46.13 \%$ & 24.29 & \multirow[t]{5}{*}{6} & 6 & $47.30 \%$ & 301.83 & $47.30 \%$ & 23.29 \\
\hline & 8 & $56.52 \%$ & 134.02 & $56.52 \%$ & 28.41 & & 8 & $57.67 \%$ & 91.55 & $57.67 \%$ & 28.02 & & 8 & $57.18 \%$ & 88.56 & $57.18 \%$ & 28.35 \\
\hline & 10 & $65.70 \%$ & 50.68 & $65.70 \%$ & 35.00 & & 10 & $64.64 \%$ & 57.96 & $64.64 \%$ & 36.59 & & 10 & $64.49 \%$ & 66.17 & $64.49 \%$ & 35.08 \\
\hline & 12 & $72.32 \%$ & 10.21 & $72.32 \%$ & 38.56 & & 12 & $70.65 \%$ & 9.02 & $70.65 \%$ & 44.01 & & 12 & $69.90 \%$ & 14.02 & $69.90 \%$ & 46.31 \\
\hline & 14 & $76.10 \%$ & 7.19 & $76.10 \%$ & 44.89 & & 14 & $74.08 \%$ & 8.24 & $74.08 \%$ & 46.25 & & 14 & $74.37 \%$ & 7.05 & $74.37 \%$ & 47.18 \\
\hline \multirow[t]{5}{*}{8} & 6 & $35.61 \%$ & 162.22 & $35.61 \%$ & 21.03 & \multirow[t]{5}{*}{8} & 6 & $37.51 \%$ & 237.56 & $37.51 \%$ & 26.04 & \multirow[t]{5}{*}{8} & 6 & $42.38 \%$ & 196.42 & $42.38 \%$ & 22.15 \\
\hline & 8 & $43.86 \%$ & 121.89 & $43.86 \%$ & 28.82 & & 8 & $45.32 \%$ & 144.19 & $45.32 \%$ & 37.75 & & 8 & $48.93 \%$ & 123.28 & $48.93 \%$ & 34.53 \\
\hline & 10 & $51.07 \%$ & 84.03 & $51.07 \%$ & 34.96 & & 10 & $52.15 \%$ & 79.13 & $52.15 \%$ & 39.98 & & 10 & $55.64 \%$ & 94.55 & $55.64 \%$ & 36.95 \\
\hline & 12 & $57.28 \%$ & 37.09 & $57.28 \%$ & 40.03 & & 12 & $57.94 \%$ & 29.04 & $57.94 \%$ & 45.33 & & 12 & $61.40 \%$ & 30.73 & $61.40 \%$ & 46.17 \\
\hline & 14 & $63.24 \%$ & 7.81 & $63.24 \%$ & 45.31 & & 14 & $62.08 \%$ & 12.87 & $62.08 \%$ & 54.20 & & 14 & $66.04 \%$ & 7.95 & $66.04 \%$ & 49.83 \\
\hline \multirow[t]{5}{*}{10} & 6 & $27.57 \%$ & 155.79 & $27.57 \%$ & 21.26 & \multirow[t]{5}{*}{10} & 6 & $32.85 \%$ & 88.97 & $32.85 \%$ & 20.09 & \multirow[t]{5}{*}{10} & 6 & $37.74 \%$ & 86.03 & $37.74 \%$ & 26.05 \\
\hline & 8 & $33.84 \%$ & 158.76 & $33.84 \%$ & 30.11 & & 8 & $40.38 \%$ & 98.69 & $40.38 \%$ & 31.00 & & 8 & $43.36 \%$ & 111.82 & $43.36 \%$ & 32.55 \\
\hline & 10 & $39.64 \%$ & 64.13 & $39.64 \%$ & 39.53 & & 10 & $46.82 \%$ & 45.58 & $46.82 \%$ & 32.49 & & 10 & $48.41 \%$ & 75.64 & $48.41 \%$ & 40.13 \\
\hline & 12 & $45.03 \%$ & 39.82 & $45.03 \%$ & 42.80 & & 12 & $51.77 \%$ & 11.52 & $51.77 \%$ & 39.47 & & 12 & $52.74 \%$ & 30.73 & $52.74 \%$ & 46.89 \\
\hline & 14 & $49.37 \%$ & 13.77 & $49.37 \%$ & 47.00 & & 14 & $54.87 \%$ & 9.09 & $54.87 \%$ & 44.93 & & 14 & $56.41 \%$ & 11.46 & $56.41 \%$ & 49.16 \\
\hline \multirow[t]{5}{*}{12} & 6 & $20.63 \%$ & 112.27 & $20.63 \%$ & 23.49 & \multirow[t]{5}{*}{12} & 6 & $25.03 \%$ & 177.42 & $25.03 \%$ & 21.13 & 12 & 6 & $28.74 \%$ & 231.91 & $28.74 \%$ & 25.74 \\
\hline & 8 & $26.75 \%$ & 64.53 & $26.75 \%$ & 31.98 & & 8 & $30.67 \%$ & 60.04 & $30.67 \%$ & 31.47 & & 8 & $33.24 \%$ & 136.65 & $33.24 \%$ & 30.12 \\
\hline & 10 & $31.95 \%$ & 33.96 & $31.95 \%$ & 34.81 & & 10 & $34.67 \%$ & 43.86 & $34.67 \%$ & 40.89 & & 10 & $37.50 \%$ & 74.81 & $37.50 \%$ & 36.18 \\
\hline & 12 & $36.59 \%$ & 15.95 & $36.59 \%$ & 40.02 & & 12 & $37.70 \%$ & 30.75 & $37.70 \%$ & 50.16 & & 12 & $40.92 \%$ & 46.94 & $40.92 \%$ & 42.56 \\
\hline & 14 & $39.73 \%$ & 8.06 & $39.73 \%$ & 42.19 & & 14 & $40.02 \%$ & 22.60 & $40.02 \%$ & 51.83 & & 14 & $44.07 \%$ & 16.86 & $44.07 \%$ & 46.98 \\
\hline 14 & 6 & $18.16 \%$ & 39.65 & $18.16 \%$ & 24.61 & 14 & 6 & $22.32 \%$ & 60.74 & $22.32 \%$ & 27.02 & 14 & 6 & $24.71 \%$ & 104.80 & $24.71 \%$ & 22.98 \\
\hline & 8 & $22.82 \%$ & 28.22 & $22.82 \%$ & 35.38 & & 8 & $27.35 \%$ & 46.29 & $27.35 \%$ & 33.17 & & 8 & $29.13 \%$ & 53.78 & $29.13 \%$ & 29.01 \\
\hline & 10 & $27.07 \%$ & 12.62 & $27.07 \%$ & 34.09 & & 10 & $30.50 \%$ & 27.30 & $30.50 \%$ & 40.21 & & 10 & $32.48 \%$ & 33.16 & $32.48 \%$ & 33.29 \\
\hline & 12 & $30.02 \%$ & 15.14 & $30.02 \%$ & 44.14 & & 12 & $32.93 \%$ & 24.36 & $32.93 \%$ & 48.85 & & 12 & $35.42 \%$ & 17.62 & $35.42 \%$ & 42.34 \\
\hline & 14 & $31.93 \%$ & 8.36 & $31.93 \%$ & 48.90 & & 14 & $34.94 \%$ & 15.24 & $34.94 \%$ & 61.97 & & 14 & $37.19 \%$ & 12.96 & $37.19 \%$ & 46.39 \\
\hline Ave & age & $41.92 \%$ & 65.82 & $41.92 \%$ & 35.09 & Ave & age & $44.43 \%$ & 76.14 & $44.43 \%$ & 38.28 & Ave & age & $46.78 \%$ & 79.03 & $46.78 \%$ & 36.80 \\
\hline
\end{tabular}

Table 12

Computational analysis results for single allocation $(r \mid p)$ hub-centroid problem with small instances.

\begin{tabular}{|c|c|c|c|c|c|c|c|c|c|c|c|c|c|}
\hline \multicolumn{7}{|c|}{$\alpha=0.6$} & \multicolumn{7}{|c|}{$\alpha=0.8$} \\
\hline \multirow[t]{2}{*}{$n$} & \multirow[t]{2}{*}{$p$} & \multirow[t]{2}{*}{$r$} & \multicolumn{2}{|l|}{ Enumeration } & \multicolumn{2}{|l|}{ SA } & \multirow[t]{2}{*}{$\bar{n}$} & \multirow{2}{*}{$p$} & \multirow[t]{2}{*}{$r$} & \multicolumn{2}{|l|}{ Enumeration } & \multicolumn{2}{|l|}{ SA } \\
\hline & & & Follower's capture & $\mathrm{CPU}(\mathrm{s})$ & Follower's capture & $\mathrm{CPU}(\mathrm{s})$ & & & & Follower's capture & $\mathrm{CPU}(\mathrm{s})$ & Follower's capture & $\mathrm{CPU}(\mathrm{s})$ \\
\hline \multirow[t]{4}{*}{10} & 2 & 2 & $42.18 \%$ & 0.79 & $42.18 \%$ & 6.99 & 10 & 2 & 2 & $42.19 \%$ & 0.65 & $42.19 \%$ & 7.87 \\
\hline & & 3 & $60.08 \%$ & 24.47 & $60.08 \%$ & 9.96 & & & 3 & $57.10 \%$ & 36.32 & $57.10 \%$ & 10.08 \\
\hline & 3 & 2 & $30.59 \%$ & 6.17 & $30.59 \%$ & 6.87 & & 3 & 2 & $31.52 \%$ & 7.37 & $31.52 \%$ & 7.18 \\
\hline & & 3 & $43.84 \%$ & 90.65 & $43.84 \%$ & 10.15 & & & 3 & $43.33 \%$ & 94.37 & $43.33 \%$ & 9.70 \\
\hline \multirow[t]{4}{*}{15} & 2 & 2 & $45.27 \%$ & 2382.07 & $45.27 \%$ & 57.87 & 15 & 2 & 2 & $42.58 \%$ & 2491.82 & $42.58 \%$ & 55.80 \\
\hline & & 3 & time & - & $63.25 \%$ & 75.97 & & & 3 & time & - & $60.64 \%$ & 77.87 \\
\hline & 3 & 2 & memory & - & $35.02 \%$ & 51.77 & & 3 & 2 & memory & - & $36.53 \%$ & 54.43 \\
\hline & & 3 & memory & - & $52.71 \%$ & 74.50 & & & 3 & memory & - & $51.12 \%$ & 75.06 \\
\hline \multicolumn{3}{|c|}{ Average } & - & - & $46.62 \%$ & 36.76 & \multicolumn{3}{|c|}{ Average } & - & - & $45.63 \%$ & 37.25 \\
\hline
\end{tabular}

$p$ or $r$ larger than 2. In case $p=2$ and $r=3$, the solution times exceed the given time limit of $3 \mathrm{~h}$. Moreover, for the instances with $p=3$, the problem cannot be solved because of excessive memory requirements. Therefore, even for very small instances, an optimal solution of single allocation $(r \mid p)$ hub-centroid problem cannot be obtained by an enumeration algorithm. This is because of the fact that the single allocation HLP is a more difficult problem than the multiple allocation HLP. In fact, as stated in Section 1, given a fixed set of locations for the hubs, the allocation part of the problem is still NP-hard for a single allocation HLP. However, as can be seen from Table 12, the proposed SA algorithm is able to solve all the instances in very short computational times.

Results for solving the single allocation $(r \mid p)$ hub-centroid problem for the CAB and TR data sets are presented in Tables 13 and 14 , respectively. As it is only possible to solve this problem for very small instances using the enumeration algorithm, and also since the problem has not been studied in the literature prior to this work, the results obtained by the proposed SA algorithm are not compared to any other results. It should be mentioned that, after solving the bilevel problem using the proposed SA, the follower's (single level) problem was solved by CPLEX based on the obtained solution for the leader and it was observed that the SA has solved the follower's problem to optimality for all the instances. Nevertheless, the optimality of the leader's solution obtained by the SA is not proven.

It can be seen from Tables 13 and 14 that, for the CAB data set, the leader's market share has increased considerably as he/she decided based on $(r \mid p)$ hub-centroid problem rather than deciding based on the USApHMP. In case of the TR data set, the increase in the leader's capture when he/she decides in anticipation of a competition is also higher than the corresponding capture when this competition is ignored by the leader. We should also note that, as mentioned above, the optimality of the leader's solutions obtained by the SA is not proven. Therefore, if the optimal solutions for the leader are found, the corresponding capture values by the follower can even be smaller. In other words, the leader's gain as a result of solving the bilevel model can even be larger.

The results reported in Tables 13 and 14 indicate that solving the single allocation $(r \mid p)$ hub-centroid problem using the proposed SA takes longer CPU times than the time needed to solve the corresponding problem under the multiple allocation setting. This is mainly because of the fact that the single allocation HLPs are much harder to solve than their multiple allocation counterparts as in the former case the allocation of every node must be explicitly 
Table 13

Results for single allocation $(r \mid p)$ hub-centroid problem with the CAB data set.

\begin{tabular}{|c|c|c|c|c|c|c|c|}
\hline \multicolumn{4}{|c|}{$\alpha=0.6$} & \multicolumn{4}{|c|}{$\alpha=0.8$} \\
\hline \multirow[t]{2}{*}{$p$} & \multirow[t]{2}{*}{$r$} & \multicolumn{2}{|l|}{ SA } & \multirow[t]{2}{*}{$p$} & \multirow[t]{2}{*}{$r$} & \multicolumn{2}{|l|}{ SA } \\
\hline & & Follower's capture & $\mathrm{CPU}(\mathrm{s})$ & & & Follower's capture & CPU (s) \\
\hline \multirow[t]{4}{*}{2} & 2 & $52.52 \%$ & 192.51 & \multirow[t]{4}{*}{2} & 2 & $50.31 \%$ & 172.30 \\
\hline & 3 & $65.77 \%$ & 272.08 & & 3 & $63.55 \%$ & 276.78 \\
\hline & 4 & $74.29 \%$ & 325.90 & & 4 & $69.38 \%$ & 311.99 \\
\hline & 5 & $81.82 \%$ & 395.31 & & 5 & $76.13 \%$ & 379.45 \\
\hline \multirow[t]{4}{*}{3} & 2 & $38.57 \%$ & 189.75 & \multirow[t]{4}{*}{3} & 2 & $42.24 \%$ & 180.09 \\
\hline & 3 & $50.03 \%$ & 281.45 & & 3 & $50.86 \%$ & 235.51 \\
\hline & 4 & $57.88 \%$ & 319.92 & & 4 & $58.32 \%$ & 321.49 \\
\hline & 5 & $64.17 \%$ & 388.84 & & 5 & $63.84 \%$ & 367.35 \\
\hline \multirow[t]{4}{*}{4} & 2 & $28.31 \%$ & 196.18 & \multirow[t]{4}{*}{4} & 2 & $36.40 \%$ & 193.75 \\
\hline & 3 & $36.98 \%$ & 280.63 & & 3 & $43.53 \%$ & 279.80 \\
\hline & 4 & $44.39 \%$ & 334.22 & & 4 & $49.48 \%$ & 321.61 \\
\hline & 5 & $51.20 \%$ & 395.40 & & 5 & $54.88 \%$ & 395.97 \\
\hline \multirow[t]{4}{*}{5} & 2 & $20.74 \%$ & 201.43 & \multirow[t]{4}{*}{5} & 2 & $30.53 \%$ & 190.41 \\
\hline & 3 & $29.73 \%$ & 296.55 & & 3 & $37.82 \%$ & 294.98 \\
\hline & 4 & $37.32 \%$ & 328.52 & & 4 & $43.42 \%$ & 336.34 \\
\hline & 5 & $44.18 \%$ & 349.38 & & 5 & $48.30 \%$ & 401.05 \\
\hline \multicolumn{2}{|c|}{ Average } & $48.62 \%$ & 296.75 & \multicolumn{2}{|c|}{ Average } & $51.19 \%$ & 291.17 \\
\hline
\end{tabular}

Table 14

Results for single allocation $(r \mid p)$ hub-centroid problem with the TR data set.

\begin{tabular}{|c|c|c|c|c|c|c|c|c|c|c|c|}
\hline \multicolumn{4}{|c|}{$\alpha=0.6$} & \multicolumn{4}{|c|}{$\alpha=0.8$} & \multicolumn{4}{|c|}{$\alpha=0.9$} \\
\hline \multirow[t]{2}{*}{$p$} & \multirow[t]{2}{*}{$r$} & \multicolumn{2}{|l|}{ SA } & \multirow[t]{2}{*}{$p$} & \multirow[t]{2}{*}{$r$} & \multicolumn{2}{|l|}{ SA } & \multirow[t]{2}{*}{$p$} & \multirow[t]{2}{*}{$r$} & \multicolumn{2}{|l|}{ SA } \\
\hline & & Follower's capture & CPU (s) & & & Follower's capture & CPU (s) & & & Follower's capture & CPU (s) \\
\hline \multirow[t]{3}{*}{2} & 2 & $50.44 \%$ & 1653.94 & \multirow[t]{3}{*}{2} & 2 & $49.95 \%$ & 1738.26 & \multirow[t]{3}{*}{2} & 2 & $49.14 \%$ & 1729.31 \\
\hline & 3 & $63.56 \%$ & 2274.58 & & 3 & $60.12 \%$ & 2136.18 & & 3 & $55.20 \%$ & 2198.02 \\
\hline & 4 & $72.80 \%$ & 2810.82 & & 4 & $72.37 \%$ & 2503.93 & & 4 & $67.40 \%$ & 2725.88 \\
\hline \multirow{3}{*}{3} & 3 & $48.81 \%$ & 2254.56 & \multirow{3}{*}{3} & 3 & $53.31 \%$ & 2258.39 & \multirow{3}{*}{3} & 3 & $53.24 \%$ & 2199.02 \\
\hline & 4 & $58.63 \%$ & 2815.18 & & 4 & $58.14 \%$ & 2789.45 & & 4 & $57.05 \%$ & 2784.51 \\
\hline & 5 & $67.55 \%$ & 3274.46 & & 5 & $69.57 \%$ & 3290.01 & & 5 & $63.87 \%$ & 3244.91 \\
\hline \multirow[t]{3}{*}{4} & 2 & $31.57 \%$ & 1793.85 & \multirow[t]{3}{*}{4} & 2 & $34.20 \%$ & 1793.85 & \multirow[t]{3}{*}{4} & 2 & $35.05 \%$ & 1805.63 \\
\hline & 3 & $39.81 \%$ & 2425.31 & & 3 & $38.63 \%$ & 2425.31 & & 3 & $42.59 \%$ & 2319.55 \\
\hline & 4 & $46.78 \%$ & 2988.74 & & 4 & $46.61 \%$ & 2988.74 & & 4 & $49.21 \%$ & 2875.13 \\
\hline 5 & 5 & $45.04 \%$ & 3795.74 & 5 & 5 & $45.14 \%$ & 3895.00 & 5 & 5 & $45.99 \%$ & 4008.07 \\
\hline \multicolumn{2}{|c|}{ Average } & $49.62 \%$ & 2603.02 & \multicolumn{2}{|c|}{ Average } & $50.23 \%$ & 2569.17 & \multicolumn{2}{|c|}{ Average } & $50.12 \%$ & 2597.81 \\
\hline
\end{tabular}

determined by the SA algorithm while in the latter case the allocations are determined by solving simple shortest path problems once the location of hubs are fixed by the algorithm. However, it is obvious that even for the single allocation network, the time it takes to obtain the final solution by the SA algorithm is not very much as spending an average of less than $2600 \mathrm{~s}$ of CPU for solving a strategic planning problem such as the competitive HLP on a large sized data set of TR is quite reasonable.

\subsection{Optimal hub locations on map}

In this part of our computational studies we illustrate how the decisions regarding the location of hubs alter for the leader and follower as the leader makes his/her decisions with and without being aware of the follower's upcoming entrance to the market. The analysis is conducted for both single and multiple allocation networks for the TR data set. Fig. 4 depicts the optimal locations of hubs opened by the leader and the follower in a multiple allocation network with $p=3, r=3$, and $\alpha=0.9$ on the map of Turkey. The 22 candidate cities for locating the hub facilities are shown as red circles in these maps. In part (a) it is assumed that the leader ignores the competition and locates its hubs based on UMApHMP, whereas part (b) depicts the location of hubs in case the leader locates its hubs based on the solution of $(r \mid p)$ hub-centroid problem. Observe that the optimal set of hub locations when the competition is ignored by the leader are different from the optimal set of hubs when the competition is incorporated into the location problem by the leader.

Fig. 5 depicts the corresponding optimal hub locations of the leader and the follower in a single allocation network.

It can be seen from Fig. 5 that in case of the single allocation network (as in the multiple allocation network), the hubs opened by the leader and the follower are affected by either considering the competition in the decision making or not. Another interesting point is that, the two nodes 6 (Ankara) and 34 (Istanbul) are selected as hub facilities by both the leader and the follower in the solutions depicted in Fig. 5 (a) and (b), respectively.

It is shown from the above maps that the optimal set of hubs opened by the leader and the follower are different for single and multiple allocation networks. This can be regarded as a reason why both the allocation protocols need to be considered in studying the competitive hub networks. Moreover, it can be seen that the hubs are generally located in the western part of the country as the candidate cities in the western part have larger volumes of incom- 


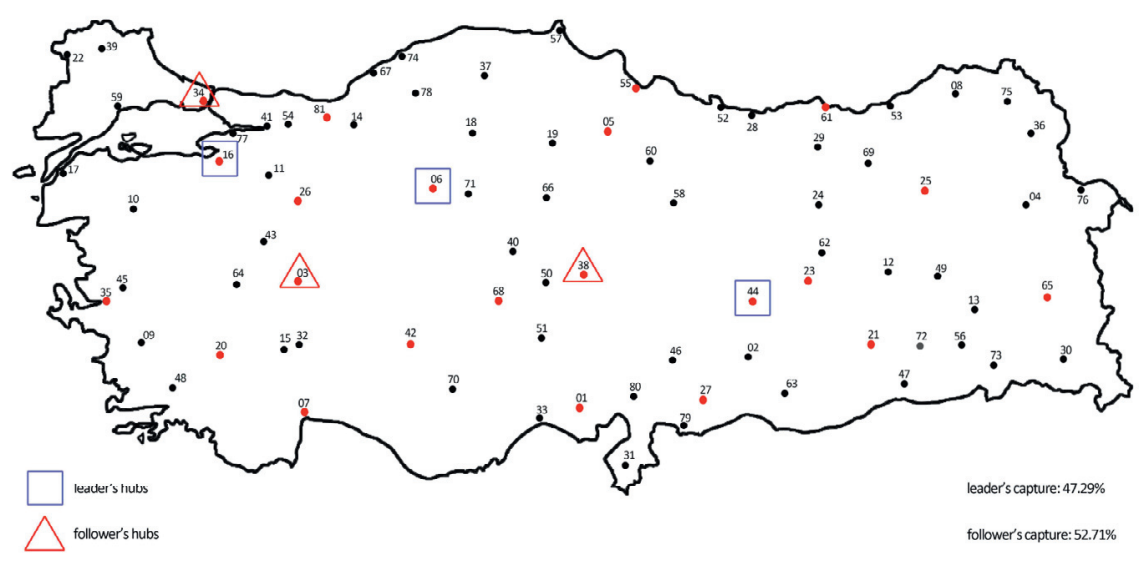

(a) leader acts based on UMApHMP

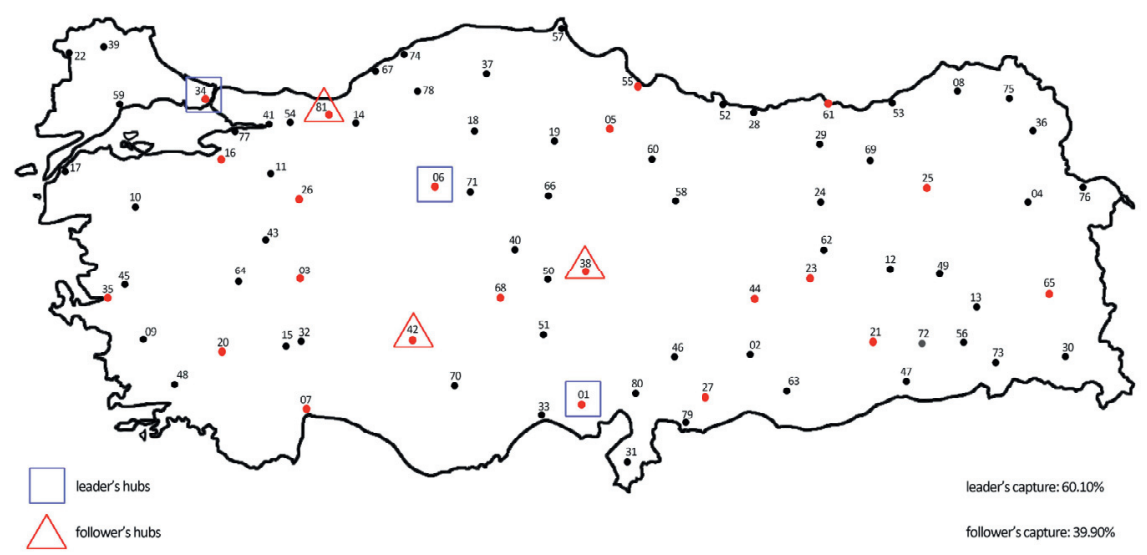

(b) leader acts based on $(r \mid p)$ hub-centroid

Fig. 4. Optimal hub locations for leader and follower with $p=r=3$ and $\alpha=0.9$ on multiple allocation network.

ing/outgoing traffic compared to the eastern cities. Note that the node 6 (Ankara) is selected as hub in all of the four maps illustrated in Figs. 4 and 5. This can be explained by central geographic location of this city within the country and also by high volume of incoming and outgoing flows to and from Ankara as the capital and the second largest city in the country. Furthermore, it can be seen that the two nodes 34 (Istanbul) and 38 (Kayseri) are selected as hubs in three of the four depicted solutions. This is mainly because Istanbul (as the largest city in the country) is the highest contributor to the total traffic volume and Kayseri has an attractive geographic location at the center of the country acting as a connector between the western and the eastern cities.

\subsection{Value of competitive solution}

Results presented in previous sub-sections reveal that when the leader acts in anticipation of follower's entrance and configures its networks based on the solution of the $(r \mid p)$ hub-centroid problem, the value of follower's capture is lower than that of the case where the leader ignores the competition and acts based on UMApHMP/USApHMP. In other words, the leader increases its market share by locating its hubs based on the solution of the bilevel (centroid) models. Increased market share creates value for the leader and we call this value as the value of competitive solution (VCS). We formally define the VCS as the extra percent market share gained by the leader when the competition is taken into account in his/her decisions compared to the case in which the leader ignores the competition and makes its decisions solely based on the cost criterion. The VCS values for different instances of the $C A B$ data set with multiple and single allocation networks are presented in Table 15.

Observe from the Table 15 that for the CAB data set, the VCS values are positive for most of the instances. It means that for most of the cases, acting based on the solution of the centroid problem creates strictly positive value for the leader. Note also that the average VCS values are significantly larger for single allocation network compared to the multiple allocation network. In other words, for the $C A B$ data set, the benefit of using the competitive model for the leader is higher when the underlying network is a single allocation network. However, even for multiple allocation case, the 


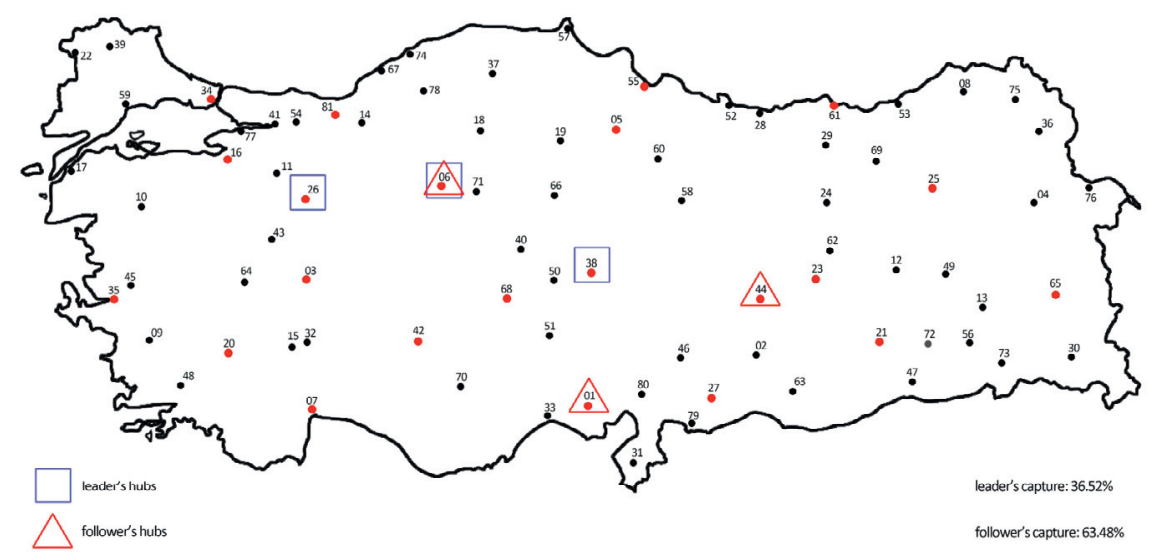

(a) leader acts based on USApHMP

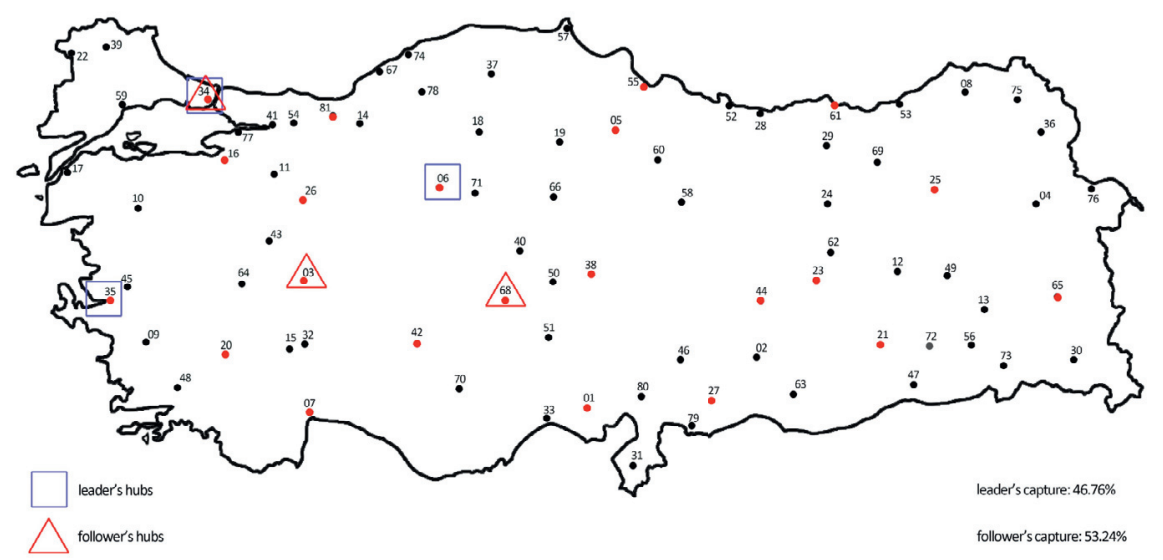

(b) leader acts based on $(r \mid p)$ hub-centroid

Fig. 5. Optimal hub locations for leader and follower with $p=r=3$ and $\alpha=0.9$ on single allocation network.

VCS is a significant value around $5 \%$ as preventing an extra $5 \%$ of market share to be captured by the follower is definitely of great importance for the leader. The VCS values for different instances of the TR data set with multiple and single allocation networks are reported in Table 16.

It can be seen that the VCS for the TR data set is in general less than that of the $C A B$ data set. One reason for this observation might be the fact that in the TR data set, the number of candidate nodes for locating hub facilities is limited $(|H|=22)$. This limited set of candidate hub nodes make the action space of the decision makers limited, which in turn results in less difference between the set of leader's hubs when he/she decides based on either of $p$-hub median problem or $(r \mid p)$ hubcentroid problem. As a result, the VCS values are rather small in case of the TR data set as compared to the case of the CAB data set. However, in both the data sets, it can be seen that the VCS generally gets larger as the value of the discount factor increases.

\subsection{Considering more general capture mechanisms}

So far, in this paper, we have assumed that the customers behave based on a all-or-nothing (or binary) mechanism towards selecting from among competing firms in the market. In other words, if a firm (e.g., the follower) offers a service with a slightly lower cost than that of the other firm (i.e., the leader) for a specific O/D pair, then all the demand for that O/D pair is assumed to fully patronize the former. This assumption may seem unrealistic to some extent as deciding on the service providers is normally based on other factors such as brand loyalty, reputation, etc. Therefore, a more realistic assumption is that the follower can capture only a percentage of the traffic volume if it's cost is not significantly less than that of the leader. This assumption has been used in some competitive HLP works (see Marianov et al., 1999; Sasaki et al., 2014; Wagner, 2008, among others). To this end, we need to slightly change the definition of the covering parameter, $a_{i j}^{k m}$, which previously was a binary parameter as defined in (4). Instead of this binary parameter, we use a five-level covering parameter 
Table 15

Value of competitive solution with the CAB data set.

\begin{tabular}{|c|c|c|c|c|c|c|c|}
\hline \multicolumn{4}{|c|}{$\alpha=0.6$} & \multicolumn{4}{|c|}{$\alpha=0.8$} \\
\hline \multirow[t]{2}{*}{$p$} & \multirow[t]{2}{*}{$r$} & \multicolumn{2}{|l|}{ VCS } & \multirow[t]{2}{*}{$p$} & \multirow[t]{2}{*}{$r$} & \multicolumn{2}{|l|}{ VCS } \\
\hline & & Mult. Alloc. & Sing. Alloc. & & & Mult. Alloc. & Sing. Alloc. \\
\hline \multirow[t]{4}{*}{2} & 2 & $19.48 \%$ & $9.59 \%$ & \multirow[t]{4}{*}{2} & 2 & $22.16 \%$ & $10.94 \%$ \\
\hline & 3 & $13.89 \%$ & $12.69 \%$ & & 3 & $14.60 \%$ & $6.86 \%$ \\
\hline & 4 & $12.33 \%$ & $14.61 \%$ & & 4 & $9.95 \%$ & $11.47 \%$ \\
\hline & 5 & $8.86 \%$ & $11.67 \%$ & & 5 & $8.41 \%$ & $13.11 \%$ \\
\hline \multirow[t]{4}{*}{3} & 2 & $0.10 \%$ & $3.99 \%$ & \multirow[t]{4}{*}{3} & 2 & $0.00 \%$ & $4.03 \%$ \\
\hline & 3 & $0.00 \%$ & $8.97 \%$ & & 3 & $0.05 \%$ & $10.00 \%$ \\
\hline & 4 & $0.00 \%$ & $13.44 \%$ & & 4 & $0.00 \%$ & $14.72 \%$ \\
\hline & 5 & $0.00 \%$ & $19.24 \%$ & & 5 & $0.00 \%$ & $19.15 \%$ \\
\hline \multirow[t]{4}{*}{4} & 2 & $0.98 \%$ & $0.22 \%$ & \multirow[t]{4}{*}{4} & 2 & $0.00 \%$ & $5.02 \%$ \\
\hline & 3 & $0.00 \%$ & $0.00 \%$ & & 3 & $1.99 \%$ & $5.51 \%$ \\
\hline & 4 & $0.00 \%$ & $0.00 \%$ & & 4 & $3.71 \%$ & $7.75 \%$ \\
\hline & 5 & $0.00 \%$ & $0.53 \%$ & & 5 & $3.36 \%$ & $9.82 \%$ \\
\hline \multirow[t]{4}{*}{5} & 2 & $4.35 \%$ & $5.42 \%$ & \multirow[t]{4}{*}{5} & 2 & $2.90 \%$ & $8.59 \%$ \\
\hline & 3 & $4.41 \%$ & $3.89 \%$ & & 3 & $5.89 \%$ & $9.10 \%$ \\
\hline & 4 & $3.14 \%$ & $2.11 \%$ & & 4 & $5.15 \%$ & $9.93 \%$ \\
\hline & 5 & $2.75 \%$ & $0.83 \%$ & & 5 & $5.77 \%$ & $11.22 \%$ \\
\hline \multicolumn{2}{|c|}{ Average } & $4.39 \%$ & $6.70 \%$ & \multicolumn{2}{|c|}{ Average } & $5.25 \%$ & $9.82 \%$ \\
\hline
\end{tabular}

that allows each firm to capture $0 \%, 25 \%, 50 \%, 75 \%$ or $100 \%$ of the demand volume on each $\mathrm{O} / \mathrm{D}$ route based on the cost of the provided service. Hence, for all $i, j \in N$ and $k, m \in H$, we define the fivelevel covering parameter as: general capture mechanism produces solutions for which the market shares for the leader and the follower are more balanced compared to the case of binary capture mechanism. For example, the follower's capture for the medianoid problem with $\alpha=0.6$ has increased by $4.74 \%$ after using the general capture mechanism. The corresponding increase for $\alpha=0.8$ is $4.87 \%$. In case of the centroid problem, the corresponding increases in the follower's capture are $6.26 \%$ and $7.37 \%$ for $\alpha=0.6$ and 0.8 , respectively. Another point of interest is that when the number of hubs to be located by the follower is the same as the number of leader's hubs $(r=p)$, the follower can capture at least $50 \%$ of the market as it can locate its hubs exactly on the same cities of the leader's hubs. Based on the above discussion, it is expected that in case of the five-level capture mechanism, the value of competitive solution (VCS) be relatively small compared to the case of the binary capture mechanism which can be seen in Table 17 .

The results for the single allocation problem under the general capture mechanism with the CAB data set are given in Table 18 .

It can be seen from Table 18 that the results obtained for the single allocation problem are more or less similar to those of the multiple allocation problem in that the market is divided in a more balanced fashion between the two firms compared to the case of the binary capture mechanism and the VCS values are smaller than their counterparts for the all-or-nothing mechanism. For instance, the follower's capture for the medianoid problem with $\alpha=0.6$ and

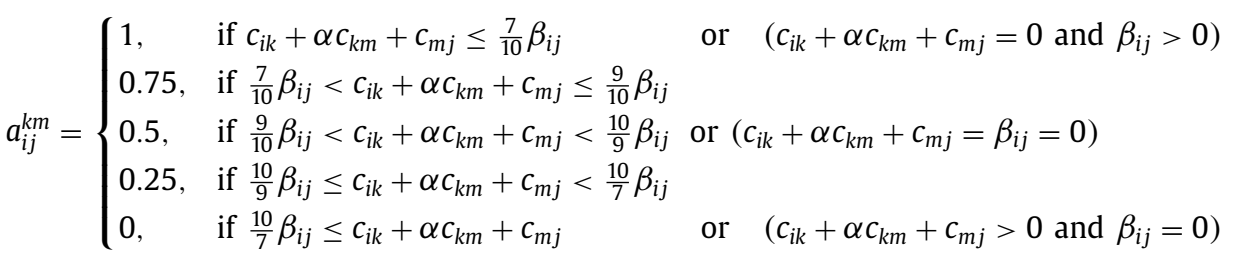

The above parameter is defined similar to the definition used in Marianov et al. (1999) and Wagner (2008). To show the effect of using the more general five-level capture mechanism on the solutions, we replaced the covering parameter previously defined as (4) by the parameter defined as (32) in our proposed mathematical formulations and solution algorithms. Table 17 shows the results obtained by solving the multiple allocation problem under the general capture mechanism with the CAB data set.

Note from Table 17 that the proposed SA algorithm is able to solve the medianoid problem to optimality in all the instances. Furthermore, the results presented in this table indicate that using the
0.8 has decreased respectively by $2.13 \%$ and $7.55 \%$ after using the general capture mechanism. In case of the centroid problem, the follower's capture has increased by $1.82 \%$ for $\alpha=0.6$, whereas it has decreased by $0.46 \%$ for $\alpha=0.8$.

As the optimality of the leader's solutions obtained by the SA is not proven in the results reported in Tables 17 and 18, the real values of the VCS can be larger than the reported values for the optimal leader's solutions. In other words, the leader's gain as a

Table 16

Value of competitive solution with the TR data set.

\begin{tabular}{|c|c|c|c|c|c|c|c|c|c|c|c|}
\hline \multicolumn{4}{|c|}{$\alpha=0.6$} & \multicolumn{4}{|c|}{$\alpha=0.8$} & \multicolumn{4}{|c|}{$\alpha=0.9$} \\
\hline \multirow[t]{2}{*}{$p$} & \multirow[t]{2}{*}{$r$} & \multicolumn{2}{|l|}{ VCS } & \multirow[t]{2}{*}{$p$} & \multirow[t]{2}{*}{$r$} & \multicolumn{2}{|l|}{ VCS } & \multirow[t]{2}{*}{$p$} & \multirow[t]{2}{*}{$r$} & \multicolumn{2}{|l|}{ VCS } \\
\hline & & Mult. Alloc. & Sing. Alloc. & & & Mult. Alloc. & Sing. Alloc. & & & Mult. Alloc. & Sing. Alloc. \\
\hline \multirow[t]{4}{*}{2} & 2 & $1.16 \%$ & $0.63 \%$ & \multirow[t]{4}{*}{2} & 2 & $3.11 \%$ & $0.57 \%$ & \multirow[t]{4}{*}{2} & 2 & $6.54 \%$ & $0.13 \%$ \\
\hline & 3 & $4.08 \%$ & $0.00 \%$ & & 3 & $2.43 \%$ & $2.55 \%$ & & 3 & $8.35 \%$ & $7.94 \%$ \\
\hline & 4 & $5.16 \%$ & $1.09 \%$ & & 4 & $2.44 \%$ & $3.37 \%$ & & 4 & $9.54 \%$ & $6.01 \%$ \\
\hline & 5 & $5.52 \%$ & $0.97 \%$ & & 5 & $6.91 \%$ & $9.33 \%$ & & 5 & $9.82 \%$ & $6.99 \%$ \\
\hline \multirow[t]{4}{*}{3} & 2 & $0.00 \%$ & $0.00 \%$ & \multirow[t]{4}{*}{3} & 2 & $0.00 \%$ & $9.87 \%$ & \multirow[t]{4}{*}{3} & 2 & $10.53 \%$ & $13.83 \%$ \\
\hline & 3 & $0.00 \%$ & $0.00 \%$ & & 3 & $0.00 \%$ & $9.10 \%$ & & 3 & $12.81 \%$ & $10.24 \%$ \\
\hline & 4 & $0.22 \%$ & $0.00 \%$ & & 4 & $0.00 \%$ & $10.29 \%$ & & 4 & $13.21 \%$ & $11.83 \%$ \\
\hline & 5 & $0.83 \%$ & $0.00 \%$ & & 5 & $0.00 \%$ & $5.16 \%$ & & 5 & $14.20 \%$ & $10.14 \%$ \\
\hline \multirow[t]{4}{*}{4} & 2 & $2.07 \%$ & $0.00 \%$ & \multirow[t]{4}{*}{4} & 2 & $0.00 \%$ & $0.00 \%$ & \multirow[t]{4}{*}{4} & 2 & $0.00 \%$ & $0.28 \%$ \\
\hline & 3 & $3.12 \%$ & $0.00 \%$ & & 3 & $0.00 \%$ & $0.00 \%$ & & 3 & $1.00 \%$ & $0.00 \%$ \\
\hline & 4 & $2.64 \%$ & $0.00 \%$ & & 4 & $0.00 \%$ & $0.00 \%$ & & 4 & $0.35 \%$ & $0.00 \%$ \\
\hline & 5 & $3.80 \%$ & $0.00 \%$ & & 5 & $0.00 \%$ & $0.00 \%$ & & 5 & $0.57 \%$ & $1.30 \%$ \\
\hline \multirow[t]{4}{*}{5} & 2 & $0.72 \%$ & $0.00 \%$ & \multirow[t]{4}{*}{5} & 2 & $0.90 \%$ & $0.00 \%$ & \multirow[t]{4}{*}{5} & 2 & $2.20 \%$ & $0.00 \%$ \\
\hline & 3 & $0.27 \%$ & $0.00 \%$ & & 3 & $2.12 \%$ & $0.00 \%$ & & 3 & $1.07 \%$ & $0.45 \%$ \\
\hline & 4 & $1.63 \%$ & $0.00 \%$ & & 4 & $3.41 \%$ & $0.00 \%$ & & 4 & $1.27 \%$ & $0.00 \%$ \\
\hline & 5 & $2.15 \%$ & $0.00 \%$ & & 5 & $2.77 \%$ & $0.00 \%$ & & 5 & $2.13 \%$ & $0.00 \%$ \\
\hline \multicolumn{2}{|c|}{ Average } & $2.08 \%$ & $0.17 \%$ & \multicolumn{2}{|c|}{ Average } & $1.50 \%$ & $3.14 \%$ & \multicolumn{2}{|c|}{ Average } & $5.84 \%$ & $4.32 \%$ \\
\hline
\end{tabular}


Table 17

Results for the general capture mechanism with the CAB data set (multiple allocation).

\begin{tabular}{|c|c|c|c|c|c|c|c|c|c|c|c|}
\hline \multicolumn{6}{|c|}{$\alpha=0.6$} & \multicolumn{6}{|c|}{$\alpha=0.8$} \\
\hline \multirow[t]{3}{*}{$p$} & \multirow[t]{3}{*}{$r$} & \multicolumn{2}{|l|}{ Medianoid } & \multirow{3}{*}{$\begin{array}{l}\text { Centroid } \\
\text { SA } \\
\text { Follower's capture }\end{array}$} & \multirow[t]{3}{*}{ VCS } & \multirow[t]{3}{*}{$p$} & \multirow[t]{3}{*}{$r$} & \multicolumn{2}{|l|}{ Medianoid } & \multirow{3}{*}{$\begin{array}{l}\text { Centroid } \\
\text { SA } \\
\text { Follower's capture }\end{array}$} & \multirow[t]{3}{*}{ VCS } \\
\hline & & CPLEX & SA & & & & & CPLEX & SA & & \\
\hline & & Follower's capture & Follower's capture & & & & & Follower's capture & Follower's capture & & \\
\hline \multirow[t]{4}{*}{2} & 2 & $59.48 \%$ & $59.48 \%$ & $50.00 \%$ & $9.48 \%$ & 2 & 2 & $58.54 \%$ & $58.54 \%$ & $50.00 \%$ & $8.54 \%$ \\
\hline & 3 & $68.82 \%$ & $68.82 \%$ & $60.04 \%$ & $8.78 \%$ & & 3 & $65.38 \%$ & $65.38 \%$ & $57.16 \%$ & $8.22 \%$ \\
\hline & 4 & $74.65 \%$ & $74.65 \%$ & $67.10 \%$ & $7.55 \%$ & & 4 & $69.87 \%$ & $69.87 \%$ & $62.74 \%$ & $7.13 \%$ \\
\hline & 5 & $79.36 \%$ & $79.36 \%$ & $72.25 \%$ & $7.11 \%$ & & 5 & $73.32 \%$ & $73.32 \%$ & $66.43 \%$ & $6.89 \%$ \\
\hline \multirow[t]{4}{*}{3} & 2 & $40.34 \%$ & $40.34 \%$ & $39.95 \%$ & $0.39 \%$ & 3 & 2 & $43.31 \%$ & $43.31 \%$ & $42.94 \%$ & $0.37 \%$ \\
\hline & 3 & $50.00 \%$ & $50.00 \%$ & $50.00 \%$ & $0.00 \%$ & & 3 & $50.06 \%$ & $50.06 \%$ & $50.00 \%$ & $0.06 \%$ \\
\hline & 4 & $57.37 \%$ & $57.37 \%$ & $57.37 \%$ & $0.00 \%$ & & 4 & $55.11 \%$ & $55.11 \%$ & $55.11 \%$ & $0.00 \%$ \\
\hline & 5 & $62.78 \%$ & $62.78 \%$ & $62.78 \%$ & $0.00 \%$ & & 5 & $59.33 \%$ & $59.33 \%$ & $59.33 \%$ & $0.00 \%$ \\
\hline \multirow[t]{4}{*}{4} & 2 & $33.84 \%$ & $33.84 \%$ & $32.89 \%$ & $0.95 \%$ & 4 & 2 & $38.31 \%$ & $38.31 \%$ & $37.23 \%$ & $1.08 \%$ \\
\hline & 3 & $43.50 \%$ & $43.50 \%$ & $42.62 \%$ & $0.88 \%$ & & 3 & $45.06 \%$ & $45.06 \%$ & $44.88 \%$ & $0.18 \%$ \\
\hline & 4 & $50.59 \%$ & $50.59 \%$ & $50.00 \%$ & $0.59 \%$ & & 4 & $50.12 \%$ & $50.12 \%$ & $50.00 \%$ & $0.12 \%$ \\
\hline & 5 & $56.50 \%$ & $56.50 \%$ & $55.82 \%$ & $0.68 \%$ & & 5 & $54.41 \%$ & $54.41 \%$ & $54.34 \%$ & $0.07 \%$ \\
\hline \multirow[t]{4}{*}{5} & 2 & $30.54 \%$ & $30.54 \%$ & $27.76 \%$ & $2.78 \%$ & 5 & 2 & $37.28 \%$ & $37.28 \%$ & $33.55 \%$ & $3.73 \%$ \\
\hline & 3 & $39.23 \%$ & $39.23 \%$ & $37.21 \%$ & $2.02 \%$ & & 3 & $43.53 \%$ & $43.53 \%$ & $40.66 \%$ & $2.87 \%$ \\
\hline & 4 & $46.52 \%$ & $46.52 \%$ & $44.17 \%$ & $2.35 \%$ & & 4 & $48.25 \%$ & $48.25 \%$ & $45.65 \%$ & $2.60 \%$ \\
\hline & 5 & $52.33 \%$ & $52.33 \%$ & $50.00 \%$ & $2.33 \%$ & & 5 & $51.96 \%$ & $51.96 \%$ & $50.00 \%$ & $1.96 \%$ \\
\hline \multicolumn{2}{|c|}{ Average } & $52.86 \%$ & $52.86 \%$ & $49.99 \%$ & $2.87 \%$ & & rage & $52.74 \%$ & $52.74 \%$ & $50.00 \%$ & $2.74 \%$ \\
\hline
\end{tabular}

Table 18

Results for the general capture mechanism with the CAB data set (single allocation).

\begin{tabular}{|c|c|c|c|c|c|c|c|c|c|c|c|}
\hline \multicolumn{6}{|c|}{$\alpha=0.6$} & \multicolumn{6}{|c|}{$\alpha=0.8$} \\
\hline \multirow[t]{3}{*}{$p$} & \multirow[t]{3}{*}{$r$} & \multicolumn{2}{|l|}{ Medianoid } & \multirow{3}{*}{$\begin{array}{l}\text { Centroid } \\
\text { SA } \\
\text { Follower's capture }\end{array}$} & \multirow[t]{3}{*}{ VCS } & \multirow[t]{3}{*}{$p$} & \multirow[t]{3}{*}{$r$} & \multicolumn{2}{|l|}{ Medianoid } & \multirow{3}{*}{$\begin{array}{l}\text { Centroid } \\
\text { SA } \\
\text { Follower's capture }\end{array}$} & \multirow[t]{3}{*}{ VCS } \\
\hline & & CPLEX & SA & & & & & CPLEX & SA & & \\
\hline & & Follower's capture & Follower's capture & & & & & Follower's capture & Follower's capture & & \\
\hline \multirow[t]{4}{*}{2} & 2 & $57.70 \%$ & $57.70 \%$ & $50.00 \%$ & $7.70 \%$ & 2 & 2 & $57.05 \%$ & $57.05 \%$ & $50.00 \%$ & $7.05 \%$ \\
\hline & 3 & $65.76 \%$ & $65.76 \%$ & $58.85 \%$ & $6.91 \%$ & & 3 & $61.74 \%$ & $61.74 \%$ & $56.26 \%$ & $5.48 \%$ \\
\hline & 4 & $72.44 \%$ & $72.44 \%$ & $66.26 \%$ & $6.18 \%$ & & 4 & $65.64 \%$ & $65.64 \%$ & $61.03 \%$ & $4.61 \%$ \\
\hline & 5 & $77.91 \%$ & $77.91 \%$ & $71.10 \%$ & $6.81 \%$ & & 5 & $69.87 \%$ & $69.87 \%$ & $64.86 \%$ & $5.01 \%$ \\
\hline \multirow[t]{4}{*}{3} & 2 & $44.10 \%$ & $44.10 \%$ & $41.14 \%$ & $2.96 \%$ & 3 & 2 & $46.89 \%$ & $46.89 \%$ & $45.18 \%$ & $1.71 \%$ \\
\hline & 3 & $51.75 \%$ & $51.75 \%$ & $50.66 \%$ & $1.09 \%$ & & 3 & $52.08 \%$ & $52.08 \%$ & $51.12 \%$ & $0.96 \%$ \\
\hline & 4 & $58.77 \%$ & $58.77 \%$ & $57.99 \%$ & $0.78 \%$ & & 4 & $56.97 \%$ & $56.97 \%$ & $56.49 \%$ & $0.48 \%$ \\
\hline & 5 & $64.96 \%$ & $64.96 \%$ & $63.33 \%$ & $1.63 \%$ & & 5 & $61.18 \%$ & $61.18 \%$ & $59.53 \%$ & $1.65 \%$ \\
\hline \multirow[t]{4}{*}{4} & 2 & $35.80 \%$ & $35.80 \%$ & $34.88 \%$ & $0.92 \%$ & 4 & 2 & $41.64 \%$ & $41.64 \%$ & $39.35 \%$ & $2.29 \%$ \\
\hline & 3 & $43.72 \%$ & $43.72 \%$ & $43.48 \%$ & $0.24 \%$ & & 3 & $47.95 \%$ & $47.95 \%$ & $46.48 \%$ & $1.47 \%$ \\
\hline & 4 & $50.76 \%$ & $50.76 \%$ & $50.75 \%$ & $0.01 \%$ & & 4 & $51.92 \%$ & $51.92 \%$ & $51.05 \%$ & $0.87 \%$ \\
\hline & 5 & $56.28 \%$ & $56.28 \%$ & $56.28 \%$ & $0.00 \%$ & & 5 & $55.88 \%$ & $55.88 \%$ & $54.98 \%$ & $0.90 \%$ \\
\hline \multirow[t]{4}{*}{5} & 2 & $32.52 \%$ & $32.52 \%$ & $29.95 \%$ & $2.57 \%$ & 5 & 2 & $39.18 \%$ & $39.18 \%$ & $36.11 \%$ & $3.07 \%$ \\
\hline & 3 & $39.89 \%$ & $39.89 \%$ & $37.05 \%$ & $2.84 \%$ & & 3 & $45.45 \%$ & $45.45 \%$ & $41.72 \%$ & $3.73 \%$ \\
\hline & 4 & $46.43 \%$ & $46.43 \%$ & $44.97 \%$ & $1.46 \%$ & & 4 & $49.22 \%$ & $49.22 \%$ & $46.78 \%$ & $2.44 \%$ \\
\hline & 5 & $52.26 \%$ & $52.26 \%$ & $50.39 \%$ & $1.87 \%$ & & 5 & $52.67 \%$ & $52.67 \%$ & $50.67 \%$ & $2.00 \%$ \\
\hline \multicolumn{2}{|c|}{ Average } & $53.19 \%$ & $53.19 \%$ & $50.44 \%$ & $2.75 \%$ & & rage & $53.46 \%$ & $53.46 \%$ & $50.73 \%$ & $2.73 \%$ \\
\hline
\end{tabular}

result of solving the bilevel model to optimality can be larger than the reported values.

\section{Conclusions}

In this research the competitive single and multiple allocation HLPs in a duopoly market are considered. Two firms sequentially decide on the location of their hubs aiming at maximizing their own market shares. It is assumed that the customers choose one firm which offers a service with lower cost. The follower's problem can be viewed as a maximal covering problem after the leader makes his/her decision. In this case, the follower aims at locating its hubs in such a way that the total captured flow (market share) is maximized. Therefore, from the leader's point of view it is important to anticipate the follower's subsequent action and incorporate it into his/her problem as a bilevel optimization program. To this end, we considered two problems for the leader and the follower: $\left(r \mid X_{p}\right)$ hub-medianoid and $(r \mid p)$ hub-centroid problems, respectively, both in single and multiple allocation networks. New bilevel MIP formulations are presented for these problems and ef- ficient simulated annealing (SA) based heuristics are proposed for solving them.

Extensive computational experiments based on two data sets of the $C A B$ and TR are conducted to analyze different properties of these problems and to evaluate the performance of the proposed SA algorithms as well as the MIP models. For all the instances with known optimal solutions from the two data sets, the proposed SA algorithms obtained the optimal solutions. However, for the bilevel single allocation problem and larger instances of the bilevel multiple allocation problem (where the optimal solutions are not known), the optimality of the solutions obtained by the SA is not proven. Furthermore, the computational results show the efficiency of the proposed algorithms in terms of CPU times. Computational experiments reveal that acting in anticipation of a possible competition creates value for the leader which we call it the value of competitive solution (VCS). Hence, solving $(r \mid p)$ hubcentroid problem is of great importance for the leader even if it requires higher amount of CPU times. We also extended the problem to accommodate a more general five-level capture mechanism in addition to the all-or-nothing (binary) mechanism. 
Further research may focus on developing exact methods such as branch-and-price or decomposition algorithms as well as different heuristics that can solve larger instances of the problems to optimality even faster. Moreover, instead of using classical HLP assumptions such as complete hub-level network or flowindependent economies of scale on only inter-hub links, more realistic assumptions like incomplete hub-level network or flowdependent scale economies on all links can be used to better reflect the real world situations.

\section{Acknowledgments}

The authors sincerely thank the anonymous referees for their valuable comments and suggestions that have helped improve the paper considerably.

\section{Appendix A}

This appendix provides linear MIP formulations for the bilevel programming problems studied in this paper. The proposed bilevel models in Section 3 are linearized using a minimax approach. To this end, we use the notations introduced in Section 3, as well as some new notations which are introduced here. We first consider the case of multiple allocation network and denote the cost of service provided by the leader for pair $i, j \in N$ by $\beta_{i j}$ as a non-negative decision variable. Moreover, let the parameter $\gamma_{i j}^{S}$ denote the cost of service provided by the follower for pair $i, j \in N$ if he/she chooses $S \subseteq H$ as the hub set. Furthermore, we define the binary decision variable $Z_{i j}^{S} \in\{0,1\}$ to indicate whether the flow from node $i \in N$ to node $j \in N$ is captured by the follower when he/she chooses $S \subseteq H$ as its hub set (by taking value of 1 ), or not (by taking value of 0 ).

The following linear MIP model solves the multiple allocation $(r \mid p)$ hub-centroid problem with an exponential number of decision variables and constraints:

$\min \theta$

s.t.: $\theta \geq \sum_{i \in N} \sum_{j \in N} w_{i j} Z_{i j}^{S} \quad \forall S \subseteq H,|S|=r$

(12) $-(14)$

$\beta_{i j} \geq \sum_{k \in H} \sum_{m \in H}\left(c_{i k}+\alpha c_{k m}+c_{m j}\right) X_{i j k m} \quad \forall i, j \in N$

$\beta_{i j}-\gamma_{i j}^{S} \leq M Z_{i j}^{S} \quad \forall i, j \in N, S \subseteq H,|S|=r$

$\theta, X_{i j k m}, \beta_{i j} \geq 0 \quad \forall i, j \in N, k, m \in H$

$Z_{i j}^{S}, Y_{k} \in\{0,1\} \quad \forall i, j \in N, k \in H, S \subseteq H,|S|=r$

The objective function (A.1) together with constraints (A.2) minimize the highest possible captured flow value by the follower in the remaining scenario. Constraints (A.4) calculate the service costs of the leader. Constraints (A.5), in which $M$ is a sufficiently large number, determine whether a flow is captured by the follower using a hub set $S \subseteq H$ of cardinality $r$ or not. More specifically, if the service cost of the follower for the flow $w_{i j}$ is smaller than the corresponding cost of the leader, $Z_{i j}^{S}$ has to take value of 1 . Otherwise, the constraint becomes redundant.

As in the case of multiple allocation models, we can convert the bilevel model for the single allocation problem to a linear MIP model using the minimax approach. To this end, let the parameter $\gamma_{i j}^{A_{S}^{f}}$ denote the cost of the service provided by the follower for pair $i, j \in N$ if he/she chooses $S \subseteq H,|S|=r$ as the hub set and $A_{S}^{f} \in \mathcal{R}_{r}(N \times H)$ as the corresponding assignment function. Also, let the binary variable $Z_{i j}^{A_{s}^{f}} \in\{0,1\}$ indicate whether the flow from node $i \in N$ to node $j \in N$ is captured by the follower for the hub set $S \subseteq H$ and the assignment function $A_{S}^{f}$. Furthermore, we define the auxiliary binary variable $V_{i j k m} \in\{0,1\}$ to take value of 1 if the nodes $i$ and $j$ are respectively assigned to the hubs $k$ and $m$ and taking 0 , otherwise. The single allocation $(r \mid p)$ hub-centroid problem can be formulated as a single level linear MIP model with an exponential number of decision variables and constraints as follows:

$\min \theta$

$$
\begin{aligned}
& \text { s.t.: } \theta \geq \sum_{i \in N} \sum_{j \in N} w_{i j} Z_{i j}^{A_{S}^{f}} \quad \forall A_{S}^{f} \in \mathcal{R}_{r}(N \times H), S \subseteq H,|S|=r \\
& (28)-(30) \\
& \beta_{i j} \geq \sum_{k \in H} \sum_{m \in H}\left(c_{i k}+\alpha c_{k m}+c_{m j}\right) V_{i j k m} \quad \forall i, j \in N \\
& \beta_{i j}-\gamma_{i j}^{A_{S}^{f}} \leq M Z_{i j}^{A_{S}^{f}} \quad \forall i, j \in N, A_{S}^{f} \in \mathcal{R}_{r}(N \times H), S \subseteq H,|S|=r
\end{aligned}
$$

$V_{i j k m} \geq Y_{i k}+Y_{j m}-1 \quad \forall i, j \in N, k, m \in H$

$\theta, \beta_{i j} \geq 0 \quad \forall i, j \in N, k, m \in H$

$Z_{i j}^{A_{s}^{f}}, Y_{i k}, V_{i j k m} \in\{0,1\}$

$\forall i, j \in N, k, m \in H, A_{S}^{f} \in \mathcal{R}_{r}(N \times H), S \subseteq H,|S|=r$

Constraints (A.11) calculate the service costs of the leader. Constraints (A.12) determine whether a flow is captured by the follower (when he/she uses the hub set $S \subseteq H$ and the assignment function $A_{S}^{f}$ ), or not. Constraints (A.13) enforce the variable $V_{i j k m}$ to be 1 , if both the variables $Y_{i k}$ and $Y_{j m}$ take value of 1 .

\section{References}

Abdinnour-Helm, S., 2001. Using simulated annealing to solve the p-hub median problem. Int. J. Phys. Distrib Logistics Manage. 31 (3), 203-220.

Abdinnour-Helm, S., Venkataramanan, M., 1998. Solution approaches to hub location problems. Ann. Oper. Res. 78, 31-50.

Abyazi-Sani, R, Ghanbari, R, 2016. An efficient tabu search for solving the uncapacitated single allocation hub location problem. Comput. Ind. Eng. 93, 99-109.

Adler, N., Smilowitz, K., 2007. Hub-and-spoke network alliances and mergers: price-location competition in the airline industry. Transport. Res. Part B 41 (4), 394-409.

Alumur, S., Kara, B.Y., 2008. Network hub location problems: the state of the art. Eur. J. Oper. Res. 190 (1), 1-21.

Bard, J.F., 1998. Practical Bilevel Optimization: Algorithms and Applications. Kluwer Academic Publishers, Dordrecht, The Netherlands.

Boland, N., Krishnamoorthy, M., Ernst, A.T., Ebery, J., 2004. Preprocessing and cutting for multiple allocation hub location problems. Eur. J. Oper. Res. 155 (3), 638-653.

Calik, H., Alumur, S.A., Kara, B.Y., Karasan, O.E., 2009. A tabu-search based heuristic for the hub covering problem over incomplete hub networks. Comput. Oper. Res. 36 (12), 3088-3096.

Campbell, J.F., 1994. Integer programming formulations of discrete hub location problems. Eur J Oper Res 72 (2), 387-405.

Campbell, J.F., 1996. Hub location and the p-hub median problem. Oper. Res. 44 (6), 923-935. 
Campbell, J.F., 2013. Modeling economies of scale in transportation hub networks. In: Proceedings of the 46th Hawaii International Conference on System Sciences (HICSS), IEEE, pp. 1154-1163.

Campbell, J.F., O'Kelly, M.E., 2012. Twenty-five years of hub location research. Transport. Sci. 46 (2), 153-169.

Černỳ, V., 1985. Thermodynamical approach to the traveling salesman problem: an efficient simulation algorithm. J. Optim. Theory Appl. 45 (1), 41-51.

Chaharsooghi, S., Momayezi, F., Ghaffarinasab, N., 2017. An adaptive large neighborhood search heuristic for solving the reliable multiple allocation hub location problem under hub disruptions. Int. J. Ind. Eng. Comput. 8 (2), 191-202.

Chen, J.-F., 2007. A hybrid heuristic for the uncapacitated single allocation hub location problem. Omega 35 (2), 211-220.

Contreras, I., 2015. Hub Location Problems. Springer International Publishing, Cham, pp. 311-344.

Contreras, I., Cordeau, J.-F., Laporte, G., 2011. Benders decomposition for large-scale uncapacitated hub location. Oper. Res. 59 (6), 1477-1490.

Dempe, S., 2002. Foundations of Bilevel Programming. Springer Science \& Business Media.

Drezner, T., Drezner, Z., Kalczynski, P., 2015. A leader-follower model for discrete competitive facility location. Comput. Oper. Res. 64, 51-59.

Eiselt, H., Marianov, V., Drezner, T., 2015. Competitive location models. In: Laporte, G., Nickel, S., Saldanha da Gama, F. (Eds.), Location Science. Springer International Publishing, pp. 365-398.

Eiselt, H.A., Marianov, V., 2009. A conditional p-hub location problem with attraction functions. Comput. Oper. Res. 36 (12), 3128-3135.

Ernst, A.T., Krishnamoorthy, M., 1996. Efficient algorithms for the uncapacitated single allocation p-hub median problem. Location Sci. 4 (3), 139-154.

Ernst, A.T., Krishnamoorthy, M., 1998. Exact and heuristic algorithms for the uncapacitated multiple allocation p-hub median problem. Eur. J. Oper. Res. 104 (1), $100-112$.

Ernst, A.T., Krishnamoorthy, M., 1999. Solution algorithms for the capacitated single allocation hub location problem. Ann. Oper. Res. 86, 141-159.

Farahani, R.Z., Hekmatfar, M., Arabani, A.B., Nikbakhsh, E., 2013. Hub location problems: a review of models, classification, solution techniques, and applications. Comput. Ind. Eng. 64 (4), 1096-1109.

Fernández, P., Pelegrín, B., Lančinskas, A., Žilinskas, J., 2017. New heuristic algorithms for discrete competitive location problems with binary and partially binary customer behavior. Comput. Oper. Res. 79, 12-18.

Gelareh, S., Nickel, S., Pisinger, D., 2010. Liner shipping hub network design in a competitive environment. Transport. Res. Part E 46 (6), 991-1004.

Jabalameli, M.S., Barzinpour, F., Saboury, A., Ghaffari-Nasab, N., 2012. A simulated annealing-based heuristic for the single allocation maximal covering hub location problem. Int. J. Metaheuristics 2 (1), 15-37.

Kara, B.Y., 1999. Modeling and Analysis of Issues in Hub Location Problem. Bilkent University Ph.D. thesis.
Kirkpatrick, S., Vecchi, M.P., et al., 1983. Optimization by simmulated annealing. Science 220 (4598), 671-680.

Kress, D., Pesch, E., 2012. Sequential competitive location on networks. Eur. J. Oper. Res. 217 (3), 483-499.

Kucukaydin, H., Aras, N., Altinel, I.K., 2012. A leader-follower game in competitive facility location. Comput. Oper. Res. 39 (2), 437-448.

Lin, C.-C., Lee, S.-C., 2010. The competition game on hub network design. Transport. Res. Part B 44 (4), 618-629.

Lüer-Villagra, A., Marianov, V., 2013. A competitive hub location and pricing problem. Eur. J. Oper. Res. 231 (3), 734-744.

Mahmutogullari, A.I., Kara, B.Y., 2016. Hub location under competition. Eur. J. Oper. Res. 250 (1), 214-225

Marianov, V., Serra, D., ReVelle, C., 1999. Location of hubs in a competitive environment. Eur. J. Oper. Res. 114 (2), 363-371.

Metropolis, N., Rosenbluth, A.W., Rosenbluth, M.N., Teller, A.H., Teller, E., 1953. Equation of state calculations by fast computing machines. J. Chem. Phys. 21 (6), 1087-1092.

O'Kelly, M.E., 1986. The location of interacting hub facilities. Transport. Sci. 20 (2), 92-106.

O'Kelly, M.E., 1987. A quadratic integer program for the location of interacting hub facilities. Eur. J. Oper. Res. 32 (3), 393-404.

Peker, M., Kara, B.Y., 2015. The p-hub maximal covering problem and extensions for gradual decay functions. Omega 54, 158-172.

Sasaki, M., 2005. Hub network design model in a competitive environment with flow threshold. J. Oper. Res. Soc. Jpn. 48 (2), 158-171.

Sasaki, M., Campbell, J.F., Krishnamoorthy, M., Ernst, A.T., 2014. A stackelberg hub arc location model for a competitive environment. Comput. Oper. Res. 47, 27-41.

Sasaki, M., Fukushima, M., 2001. Stackelberg hub location problem. J. Oper. Res. Soc Jpn. 44 (4), 390-405.

Silva, M.R., Cunha, C.B., 2009. New simple and efficient heuristics for the uncapacitated single allocation hub location problem. Comput. Oper. Res. 36 (12), 3152-3165.

Silva, M.R., Cunha, C.B., 2017. A tabu search heuristic for the uncapacitated single allocation p-hub maximal covering problem. Eur. J. Oper. Res. 262 (3), 954-965.

Skorin-Kapov, D., Skorin-Kapov, J., 1994. On tabu search for the location of interacting hub facilities. Eur. J. Oper. Res. 73 (3), 502-509.

Tan, P.Z., Kara, B.Y., 2007. A hub covering model for cargo delivery systems. Networks 49 (1), 28-39.

Topcuoglu, H., Corut, F., Ermis, M., Yilmaz, G., 2005. Solving the uncapacitated hub location problem using genetic algorithms. Comput. Oper. Res. 32 (4), 967-984.

Wagner, B., 2008. A note on location of hubs in a competitive environment. Eur. J. Oper. Res. 184 (1), 57-62. 